\title{
Virtual resolutions for a product of projective spaces
}

\author{
Christine Berkesch, Daniel Erman and Gregory G. Smith
}

\begin{abstract}
Syzygies capture intricate geometric properties of a subvariety in projective space. However, when the ambient space is a product of projective spaces or a more general smooth projective toric variety, minimal free resolutions over the Cox ring are too long and contain many geometrically superfluous summands. In this paper, we construct some much shorter free complexes that better encode the geometry.
\end{abstract}

\section{Introduction}

The geometric and algebraic sources of locally free resolutions have complementary advantages. To see the differences, consider a smooth projective toric variety $X$ together with its $\operatorname{Pic}(X)$ graded Cox ring $S$. The local version of the Hilbert syzygy theorem implies that any coherent $\mathcal{O}_{X}$-module admits a locally free resolution of length at most $\operatorname{dim} X$; see [Har77, Exercise III.6.9]. The global version of the Hilbert syzygy theorem implies that every saturated module over the polynomial ring $S$ has a minimal free resolution of length at most $\operatorname{dim} S-1$, so any coherent $\mathcal{O}_{X}$-module has a locally free resolution of the same length; see [Cox95, Proposition 3.1]. Unlike the geometric approach, this algebraic method involves only vector bundles that are a direct sum of line bundles. When $X$ is projective space, these geometric and algebraic constructions usually coincide. However, when the Picard number of $X$ is greater than 1, the locally free resolutions arising from the minimal free resolution of an $S$-module are longer, and typically much longer, than their geometric counterparts.

To enjoy the best of both worlds, we focus on a more flexible algebraic source for locally free resolutions. The following definition, beyond providing concise terminology, highlights this source.

Definition 1.1. A free complex $F:=\left[F_{0} \longleftarrow F_{1} \longleftarrow F_{2} \longleftarrow \cdots\right]$ of Pic $(X)$-graded $S$-modules is called a virtual resolution of a $\operatorname{Pic}(X)$-graded $S$-module $M$ if the corresponding complex $\widetilde{F}$ of vector bundles on $X$ is a locally free resolution of the sheaf $\widetilde{M}$.

In other words, a virtual resolution is a free complex of $S$-modules whose higher homology groups are supported on the irrelevant ideal of $X$. The benefits of allowing a limited amount of homology are already present in other parts of commutative algebra including almost ring theory [GR03], where one accepts homology annihilated by a given idempotent ideal, and phantom

Received 31 May 2017, accepted in final form 24 June 2019.

2010 Mathematics Subject Classification 13D02, 14M25, 14F05.

Keywords: toric varieties, free resolutions, vector bundles, deformation theory.

This journal is (C) Foundation Compositio Mathematica 2020. This article is distributed with Open Access under the terms of the Creative Commons Attribution Non-Commercial License, which permits non-commercial reuse, distribution, and reproduction in any medium, provided that the original work is properly cited. For commercial re-use, please contact the Foundation Compositio Mathematica.

The first author was partially supported by the NSF Grant DMS-1440537, the second author was partially supported by the NSF Grants DMS-1302057 and DMS-1601619, and the third author was partially supported by the NSERC. 


\section{VIRTUAL RESOLUTIONS}

homology [HH93], where one admits cycles that are in the tight closure of the boundaries. We describe a few different, and generally incomparable, processes for creating virtual resolutions.

For projective space, minimal free resolutions are important in the study of points [GGP95, EP99], curves [Voi02, EL15], surfaces [GP99, DS00], and moduli spaces [Far09, DFS16]. Our overarching goal is to demonstrate that the right analogues for subschemes in a smooth complete toric variety use virtual resolutions rather than minimal free resolutions. This distinction is not apparent on projective space because the new intersection theorem [Rob87] establishes that a free complex with finite-length higher homology groups has to be at least as long as the minimal free resolution. For other toric varieties such as products of projective spaces, allowing irrelevant homology may yield simpler complexes; see Example 1.4.

Throughout this paper, we write $\mathbb{P}^{n}:=\mathbb{P}^{n_{1}} \times \mathbb{P}^{n_{2}} \times \cdots \times \mathbb{P}^{n_{r}}$ for the product of projective spaces with dimension vector $\boldsymbol{n}:=\left(n_{1}, n_{2}, \ldots, n_{r}\right) \in \mathbb{N}^{r}$ over a field $\mathbb{k}$. The polynomial ring $S:=\mathbb{k}\left[x_{i, j}: 1 \leqslant i \leqslant r, 0 \leqslant j \leqslant n_{i}\right]$ is the Cox ring of $\mathbb{P}^{n}$, and $B:=\bigcap_{i=1}^{r}\left\langle x_{i, 0}, x_{i, 1}, \ldots, x_{i, n_{i}}\right\rangle$ is its irrelevant ideal. We identify the Picard group of $\mathbb{P}^{n}$ with $\mathbb{Z}^{r}$ and partially order the elements via their components. If $\boldsymbol{e}_{1}, \boldsymbol{e}_{2}, \ldots, \boldsymbol{e}_{r}$ is the standard basis of $\mathbb{Z}^{r}$, then the polynomial ring $S$ has the $\mathbb{Z}^{r}$-grading induced by $\operatorname{deg}\left(x_{i, j}\right):=\boldsymbol{e}_{i}$. We first re-prove the existence of short virtual resolutions; compare with [EES15, Corollary 2.14].

Proposition 1.2. Every finitely generated $\mathbb{Z}^{r}$-graded $B$-saturated $S$-module has a virtual resolution of length at most $|\boldsymbol{n}|:=n_{1}+n_{2}+\cdots+n_{r}=\operatorname{dim} \mathbb{P}^{\boldsymbol{n}}$.

Since $\operatorname{dim} S-\operatorname{dim} \mathbb{P}^{n}=r$, we see that a minimal free resolution can be arbitrarily long when compared with a virtual resolution. A proof of Proposition 1.2, which relies on a locally free resolution of the structure sheaf for the diagonal embedding $\mathbb{P}^{n} \hookrightarrow \mathbb{P}^{n} \times \mathbb{P}^{n}$, appears in Section 2.

Besides having shorter representatives, virtual resolutions also exhibit a closer relationship with Castelnuovo-Mumford regularity than minimal free resolutions. On projective space, CastelnuovoMumford regularity has two equivalent descriptions: one arising from the vanishing of sheaf cohomology and another arising from the Betti numbers in a minimal free resolutions. However, on more general toric varieties, the multigraded Castelnuovo-Mumford regularity is not determined by a minimal free resolution; see [MS04, Theorem 1.5] or [BC17, Theorem 4.7]. From this perspective, we demonstrate that virtual resolutions improve on minimal free resolutions in two ways. First, Theorem 2.9 proves that the set of virtual resolutions of a module determines its multigraded Castelnuovo-Mumford regularity. Second, the next theorem, from Section 3, demonstrates how to use regularity to extract a virtual resolution from a minimal free resolution.

TheOREm 1.3. Let $M$ be a finitely generated $\mathbb{Z}^{r}$-graded $B$-saturated $S$-module that is $\boldsymbol{d}$-regular. If $G$ is the free subcomplex of a minimal free resolution of $M$ consisting of all summands generated in degree at most $\boldsymbol{d}+\boldsymbol{n}$, then $G$ is a virtual resolution of $M$.

This subcomplex is seldom a resolution. For convenience, we refer to the free complex $G$ as the virtual resolution of the pair $(M, \boldsymbol{d})$. Algorithm 3.4 shows that it can be constructed without computing the entire minimal free resolution.

Our first example illustrates that a virtual resolution can be much shorter and much thinner than the minimal free resolution. It follows that a majority of the summands in the minimal free resolution are unneeded when building a locally free resolution of the structure sheaf. 


\section{Berkesch, D. Erman and G.G. Smith}

Example 1.4. A hyperelliptic curve $C$ of genus 4 can be embedded as a curve of bidegree $(2,8)$ in $\mathbb{P}^{1} \times \mathbb{P}^{2}$; see [Har77, Theorem IV.5.4]. For instance, the $B$-saturated $S$-ideal $I$ generated by

$$
\begin{aligned}
& x_{1,1}^{3} x_{2,0}-x_{1,1}^{3} x_{2,1}+x_{1,0}^{3} x_{2,2}, \\
& x_{1,0}^{2} x_{2,0}^{2}+x_{1,1}^{2} x_{2,1}^{2}+x_{1,0} x_{1,1} x_{2,2}^{2}, \\
& x_{1,1}^{2} x_{2,0}^{3}-x_{1,1}^{2} x_{2,0}^{2} x_{2,1}-x_{1,0} x_{1,1} x_{2,1}^{2} x_{2,2}-x_{1,0}^{2} x_{2,2}^{3}, \\
& x_{1,0} x_{1,1} x_{2,0}^{3}+x_{1,0} x_{1,1} x_{2,0}^{2} x_{2,1}-x_{1,0}^{2} x_{2,1}^{2} x_{2,2}+x_{1,1}^{2} x_{2,0} x_{2,2}^{2}+x_{1,1}^{2} x_{2,1} x_{2,2}^{2}, \\
& x_{1,1} x_{2,0}^{3} x_{2,1}^{2}+x_{1,1} x_{2,0}^{2} x_{2,1}^{3}-x_{1,0} x_{2,1}^{4} x_{2,2}-x_{1,0} x_{2,0}^{3} x_{2,2}^{2}+x_{1,0} x_{2,0}^{2} x_{2,1} x_{2,2}^{2}-x_{1,1} x_{2,0} x_{2,2}^{4}-x_{1,1} x_{2,1} x_{2,2}^{4}, \\
& x_{1,1} x_{2,0}^{5}+x_{1,1} x_{2,0}^{4} x_{2,1}-x_{1,0} x_{2,0}^{2} x_{2,1}^{2} x_{2,2}+x_{1,1} x_{2,1}^{2} x_{2,2}^{3}+x_{1,0} x_{2,2}^{5}, \\
& x_{1,0} x_{2,0}^{5}+x_{1,0} x_{2,0}^{4} x_{2,1}+x_{1,1} x_{2,1}^{4} x_{2,2}+x_{1,1} x_{2,0}^{3} x_{2,2}^{2}+x_{1,1} x_{2,0}^{2} x_{2,1} x_{2,2}^{2}+x_{1,0} x_{2,1}^{2} x_{2,2}^{3}, \\
& x_{2,0}^{8}+2 x_{2,0}^{7} x_{2,1}+x_{2,0}^{6} x_{2,1}^{2}+x_{2,1}^{6} x_{2,2}^{2}+3 x_{2,0}^{3} x_{2,1}^{2} x_{2,2}^{3}+3 x_{2,0}^{2} x_{2,1}^{3} x_{2,2}^{3}-x_{2,0} x_{2,2}^{7}-x_{2,1} x_{2,2}^{7}
\end{aligned}
$$

defines such a curve. Macaulay2 [GS19] shows that the minimal free resolution of $S / I$ has the form

$$
\begin{aligned}
& S(-3,-1)^{1} \\
& \oplus \quad S(-3,-3)^{3}
\end{aligned}
$$

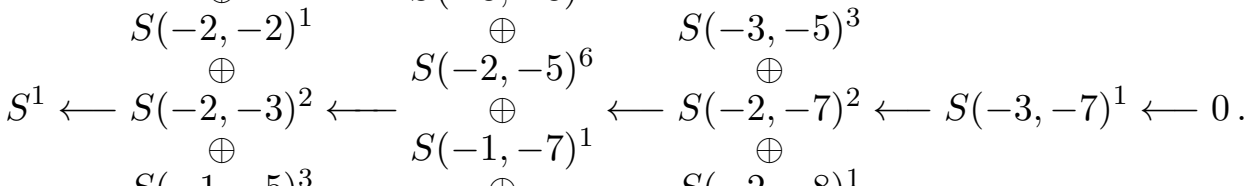

$$
\begin{aligned}
& \begin{array}{ccc}
S(-1,-5)^{3} & \multicolumn{1}{|c}{(-1,-7)^{1}} & S(-2,-8)^{1} \\
\oplus & S(-1,-8)^{2} &
\end{array} \\
& S(0,-8)^{1}
\end{aligned}
$$

Using the Riemann-Roch theorem [Har77, Theorem IV.1.3], one verifies that the module $S / I$ is $(4,2)$-regular, so the virtual resolution of the pair $(S / I,(4,2))$ has the much simpler form

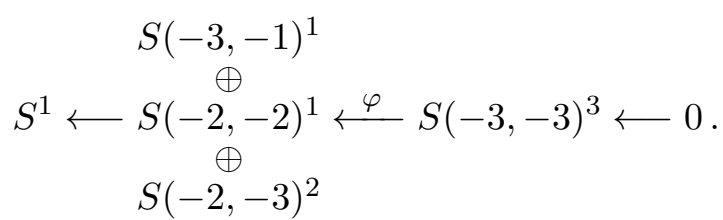

If the ideal $J \subset S$ is the image of the first map in (1.2), then we have $J=I \cap Q$ for some ideal $Q$ whose radical contains the irrelevant ideal. Using Proposition 2.5, we can even conclude that $S / J$ is Cohen-Macaulay and $J$ is the ideal of maximal minors of the $4 \times 3$ matrix

$$
\varphi:=\left[\begin{array}{ccc}
x_{2,1}^{2} & x_{2,2}^{2} & -x_{2,0}^{2} \\
-x_{1,1}\left(x_{2,0}-x_{2,1}\right) & 0 & x_{1,0} x_{2,2} \\
x_{1,0} & -x_{1,1} & 0 \\
0 & x_{1,0} & x_{1,1}
\end{array}\right] .
$$

As an initial step towards our larger goal, we formulate a novel analogue for properties of points in projective space. Although any punctual subscheme of projective space is arithmetically Cohen-Macaulay, this almost always fails for a zero-dimensional subscheme of $\mathbb{P}^{n}$; see [GV15]. However, we do obtain a short virtual resolution just by choosing an unconventional module to represent the structure sheaf on the punctual subscheme.

Theorem 1.5. Let $Z \subset \mathbb{P}^{n}$ be a zero-dimensional scheme, and let $I$ be its corresponding $B$-saturated $S$-ideal. There exists an $S$-ideal $Q$, whose radical contains $B$, such that the minimal 


\section{VIRTUAL RESOLUTIONS}

free resolution of $S /(I \cap Q)$ has length $|\boldsymbol{n}|$. In particular, the minimal free resolution of $S /(I \cap Q)$ is a virtual resolution of $S / I$.

This theorem, proven in Section 4, does not imply that $S /(I \cap Q)$ is itself Cohen-Macaulay, as the components of $Q$ will often have codimension less than $|\boldsymbol{n}|$. However, when the ambient variety is $\mathbb{P}^{1} \times \mathbb{P}^{1}$, the ring $S /(I \cap Q)$ will be Cohen-Macaulay of codimension 2 . In this case, Corollary 4.2 shows that there is a matrix whose maximal minors cut out $Z$ scheme-theoretically. Proposition 4.8 extends this to general points on any smooth toric surface.

As a second and perhaps more substantial step, we apply virtual resolutions to deformation theory. On projective space, there are three classic situations in which the particular structure of the minimal free resolution allows one to show that all deformations have the same structure: arithmetically Cohen-Macaulay subschemes of codimension 2, arithmetically Gorenstein subschemes in codimension 3, and complete intersections; see [Har10, Sections 2.8 and 2.9]. We generalize these results about unobstructed deformations in projective space as follows.

Theorem 1.6. Consider $Y \subset \mathbb{P}^{n}$, and let $I$ be the corresponding $B$-saturated $S$-ideal. Assume that the generators of $I$ have degrees $\boldsymbol{d}_{1}, \boldsymbol{d}_{2}, \ldots, \boldsymbol{d}_{s}$ and that the natural map $(S / I)_{\boldsymbol{d}_{i}} \rightarrow H^{0}\left(Y, \mathcal{O}_{Y}\left(\boldsymbol{d}_{i}\right)\right)$ is an isomorphism for all $1 \leqslant i \leqslant s$. If any one of the conditions

(i) the subscheme $Y$ has codimension 2 and there is a $\boldsymbol{d} \in \operatorname{reg}(S / I)$ such that the virtual resolution of the pair $(S / I, \boldsymbol{d})$ has length 2 ;

(ii) each factor in $\mathbb{P}^{n}$ has dimension at least 2, the subscheme $Y$ has codimension 3, and there is a $\boldsymbol{d} \in \operatorname{reg}(S / I)$ such that the virtual resolution of the pair $(S / I, \boldsymbol{d})$ is a self-dual complex (up to a twist) of length 3 ; or

(iii) there is a $\boldsymbol{d} \in \operatorname{reg}(S / I)$ such that the virtual resolution of the pair $(S / I, \boldsymbol{d})$ is a Koszul complex of length codim $Y$

holds, then the embedded deformations of $Y$ in $\mathbb{P}^{n}$ are unobstructed and the component of the multigraded Hilbert scheme of $\mathbb{P}^{\boldsymbol{n}}$ containing the point corresponding to $Y$ is unirational.

To illustrate this theorem, we can reuse the hyperelliptic curve in Example 1.4.

Example 1.7. By reinterpreting Example 1.4, we see that the hyperelliptic curve $C \subset \mathbb{P}^{1} \times \mathbb{P}^{2}$ satisfies condition (i) in Theorem 1.6. It follows that the embedded deformations of $C$ are unobstructed and the corresponding component of the multigraded Hilbert scheme of $\mathbb{P}^{1} \times \mathbb{P}^{2}$ can be given an explicit unirational parametrization by varying the entries in the $4 \times 3$ matrix $\varphi$ from (1.3).

Three other geometric applications for virtual resolutions are collected in Section 5. The first, Proposition 5.1, provides an unmixedness result for subschemes of $\mathbb{P}^{\boldsymbol{n}}$ that have a virtual resolution whose length equals its codimension. The second, Proposition 5.5, gives sharp bounds on the Castelnuovo-Mumford regularity of a tensor product of coherent $\mathcal{O}_{\mathbb{P} n}$-modules; compare with [Laz04, Proposition 1.8.8]. Lastly, Proposition 5.7 describes new vanishing results for the higher direct images of sheaves, which are optimal in many cases.

The final section presents some promising directions for future research.

Conventions. We work in the product $\mathbb{P}^{n}:=\mathbb{P}^{n_{1}} \times \mathbb{P}^{n_{2}} \times \cdots \times \mathbb{P}^{n_{r}}$ of projective spaces with dimension vector $\boldsymbol{n}:=\left(n_{1}, n_{2}, \ldots, n_{r}\right) \in \mathbb{N}^{r}$ over a field $\mathbb{k}$. Its Cox ring is the polynomial ring $S:=\mathbb{k}\left[x_{i, j}: 1 \leqslant i \leqslant r, 0 \leqslant j \leqslant n_{i}\right]$, and its irrelevant ideal is $B:=\bigcap_{i=1}^{r}\left\langle x_{i, 0}, x_{i, 1}, \ldots, x_{i, n_{i}}\right\rangle$. The Picard group of $\mathbb{P}^{n}$ is identified with $\mathbb{Z}^{r}$, and the elements are partially ordered componentwise. 


\section{Berkesch, D. Erman and G.G. Smith}

If $\boldsymbol{e}_{1}, \boldsymbol{e}_{2}, \ldots, \boldsymbol{e}_{r}$ is the standard basis of $\mathbb{Z}^{r}$, then $S$ has the $\mathbb{Z}^{r}$-grading induced by $\operatorname{deg}\left(x_{i, j}\right):=\boldsymbol{e}_{i}$. We assume that all $S$-modules are finitely generated and $\mathbb{Z}^{r}$-graded.

\section{Existence of short virtual resolutions}

This section, by proving Proposition 1.2, establishes the existence of virtual resolutions whose length is bounded above by the dimension of $\mathbb{P}^{n}$. In particular, these virtual resolutions are typically shorter than a minimal free resolution. Moreover, Proposition 2.5 shows that Proposition 1.2 provides the best possible uniform bound on the length of a virtual resolution. Exploiting multigraded Castelnuovo-Mumford regularity, we also produce short virtual resolutions where the degrees of the generators of the free modules satisfy explicit bounds. Better yet, we obtain a converse, by showing that the set of virtual resolutions of a module determine its regularity.

Our proof of Proposition 1.2 is based on a minor variation of Beilinson's resolution of the diagonal; compare with [Căl05, Proposition 3.2] or [Huy06, Lemma 8.27]. Given an $\mathcal{O}_{X_{j}}$-module $\mathcal{F}_{j}$ for all $1 \leqslant j \leqslant n$, their external tensor product is

$$
\mathcal{F}_{1} \otimes \mathcal{F}_{2} \otimes \cdots \otimes \mathcal{F}_{m}:=\left(\mathrm{p}_{1}^{*} \mathcal{F}_{1}\right) \otimes_{\mathcal{O}_{X}}\left(\mathrm{p}_{2}^{*} \mathcal{F}_{2}\right) \otimes_{\mathcal{O}_{X}} \cdots \otimes_{\mathcal{O}_{X}}\left(\mathrm{p}_{m}^{*} \mathcal{F}_{m}\right),
$$

where $\mathrm{p}_{j}$ denotes the projection map from the Cartesian product $X:=X_{1} \times X_{2} \times \cdots \times X_{m}$ to $X_{j}$. In particular, for all $\boldsymbol{u} \in \mathbb{Z}^{r}$, we have $\mathcal{O}_{\mathbb{P} \boldsymbol{n}}(\boldsymbol{u})=\mathcal{O}_{\mathbb{P}^{n_{1}}}\left(u_{1}\right) \otimes \mathcal{O}_{\mathbb{P}^{n_{2}}}\left(u_{2}\right) \otimes \cdots \otimes \mathcal{O}_{\mathbb{P}^{n} n_{r}}\left(u_{r}\right)$. With this notation, we can describe the resolution of the diagonal $\mathbb{P}^{n} \hookrightarrow \mathbb{P}^{n} \times \mathbb{P}^{n}$.

LEMMA 2.1. If $\mathcal{T}_{\mathbb{P}^{n}}^{\boldsymbol{e}_{i}}:=\mathcal{O}_{\mathbb{P}^{n_{1}}} \otimes \mathcal{O}_{\mathbb{P}^{n_{2}}} \otimes \cdots \otimes \mathcal{O}_{\mathbb{P}^{n_{i-1}}} \otimes \mathcal{T}_{\mathbb{P}^{n_{i}}} \otimes \mathcal{O}_{\mathbb{P}^{n_{i+1}}} \otimes \cdots \otimes \mathcal{O}_{\mathbb{P}^{n_{r}}}$ for $1 \leqslant i \leqslant r$, then the diagonal $\mathbb{P}^{\boldsymbol{n}} \hookrightarrow \mathbb{P}^{\boldsymbol{n}} \times \mathbb{P}^{\boldsymbol{n}}$ is the zero scheme of a global section of $\bigoplus_{i=1}^{r} \mathcal{O}_{\mathbb{P} \boldsymbol{n}}\left(\boldsymbol{e}_{i}\right) \otimes \mathcal{T}_{\mathbb{P} \boldsymbol{n}}^{\boldsymbol{e}_{i}}\left(-\boldsymbol{e}_{i}\right)$. Hence, the diagonal has a locally free resolution of the form

$$
\mathcal{O}_{\mathbb{P}^{n} \times \mathbb{P}^{n}} \longleftarrow \bigoplus_{i=1}^{r} \mathcal{O}_{\mathbb{P}^{n}}\left(-\boldsymbol{e}_{i}\right) \otimes \Omega_{\mathbb{P}^{n}}^{\boldsymbol{e}_{i}}\left(\boldsymbol{e}_{i}\right) \longleftarrow \bigoplus_{\substack{0 \leqslant \boldsymbol{u} \leqslant \boldsymbol{n} \\|\boldsymbol{u}|=2}} \mathcal{O}_{\mathbb{P}^{n}}(-\boldsymbol{u}) \otimes \Omega_{\mathbb{P}^{n}}^{\boldsymbol{u}}(\boldsymbol{u}) \leftarrow \cdots \leftarrow \mathcal{O}_{\mathbb{P}^{n}}(-\boldsymbol{n}) \otimes \Omega_{\mathbb{P}^{n}}^{\boldsymbol{n}}(\boldsymbol{n}),
$$

where $\Omega_{\mathbb{P} \boldsymbol{n}}^{a}:=\Omega_{\mathbb{P}^{n_{1}}}^{a_{1}} \otimes \Omega_{\mathbb{P}^{n_{2}}}^{a_{2}} \otimes \cdots \otimes \Omega_{\mathbb{P}_{r} n_{r}}^{a_{r}}$ is the external tensor product of the exterior powers of the cotangent bundles on the factors of $\mathbb{P}^{n}$.

Proof. For each $1 \leqslant i \leqslant r$, fix a basis $x_{i, 0}, x_{i, 1}, \ldots, x_{i, n_{i}}$ for $H^{0}\left(\mathbb{P}^{\boldsymbol{n}}, \mathcal{O}_{\mathbb{P} \boldsymbol{n}}\left(\boldsymbol{e}_{i}\right)\right)$. The Euler sequence on $\mathbb{P}^{n_{i}}$ yields

$$
0 \longleftarrow \mathcal{T}_{\mathbb{P} \boldsymbol{n}}^{\boldsymbol{e}_{i}} \longleftarrow \bigoplus_{j=0}^{n_{i}} \mathcal{O}_{\mathbb{P} \boldsymbol{n}}\left(\boldsymbol{e}_{i}\right) \stackrel{\left[x_{i, 0} x_{i, 1} \cdots x_{i, n_{i}}\right]}{\longleftarrow} \mathcal{O}_{\mathbb{P} \boldsymbol{n}} \longleftarrow 0
$$

see [CLS11, Theorem 8.1.6]. Taking into account the cohomology of line bundles on $\mathbb{P}^{n}$, the associated long exact sequence gives $H^{0}\left(\mathbb{P}^{\boldsymbol{n}}, \mathcal{T}_{\mathbb{P}^{\boldsymbol{n}}}^{\boldsymbol{e}_{i}}\left(-\boldsymbol{e}_{i}\right)\right) \cong \bigoplus_{j=0}^{n_{i}} H^{0}\left(\mathbb{P}^{\boldsymbol{n}}, \mathcal{O}_{\mathbb{P} \boldsymbol{n}}\right)$. A basis for $\bigoplus_{j=0}^{n_{i}} H^{0}\left(\mathbb{P}^{n}, \mathcal{O}_{\mathbb{P}^{n} \boldsymbol{n}}\right)$ is given by the dual basis $x_{i, 0}^{*}, x_{i, 1}^{*}, \ldots, x_{i, n_{i}}^{*}$. Let $\partial / \partial x_{i, j}$ denote the image of $x_{i, j}^{*}$ in $H^{0}\left(\mathbb{P}^{n}, \mathcal{T}_{\mathbb{P}} n\left(-\boldsymbol{e}_{i}\right)\right)$.

Consider $s \in H^{0}\left(\mathbb{P}^{\boldsymbol{n}} \times \mathbb{P}^{\boldsymbol{n}}, \bigoplus_{i=1}^{r} \mathcal{O}_{\mathbb{P} \boldsymbol{n}}\left(\boldsymbol{e}_{i}\right) \otimes \mathcal{T}_{\mathbb{P} \boldsymbol{n}}^{\boldsymbol{e}_{i}}\left(-\boldsymbol{e}_{i}\right)\right)$ given by

$$
s:=\left(\sum_{j=0}^{n_{1}} x_{1, j} \frac{\partial}{\partial y_{1, j}}, \sum_{j=0}^{n_{2}} x_{2, j} \frac{\partial}{\partial y_{2, j}}, \ldots, \sum_{j=0}^{n_{r}} x_{r, j} \frac{\partial}{\partial y_{r, j}}\right),
$$

where $x_{i, j}$ and $y_{i, j}$ are the coordinates on the first and second factor of $\mathbb{P}^{\boldsymbol{n}} \times \mathbb{P}^{\boldsymbol{n}}$ respectively. We claim that the zero scheme of $s$ equals the diagonal in $\mathbb{P}^{\boldsymbol{n}} \times \mathbb{P}^{\boldsymbol{n}}$. By symmetry, it suffices to check this on a single affine open neighborhood. If $x_{1,0} x_{2,0} \cdots x_{r, 0} \neq 0$ and $y_{1,0} y_{2,0} \cdots y_{r, 0} \neq 0$, then the 


\section{VIRTUAL RESOLUTIONS}

Euler relations yield

$$
\sum_{j=0}^{n_{i}} x_{i, j} \frac{\partial}{\partial y_{i, j}}=x_{i, 0}\left(-\frac{1}{y_{i, 0}} \sum_{j=1}^{n_{i}} y_{i, j} \frac{\partial}{\partial y_{i, j}}\right)+\sum_{j=1}^{n_{i}} x_{i, j} \frac{\partial}{\partial y_{i, j}}=\frac{1}{y_{i, 0}} \sum_{j=1}^{n_{i}}\left(x_{i, j} y_{i, 0}-x_{i, 0} y_{i, j}\right) \frac{\partial}{\partial y_{i, j}}
$$

for each $1 \leqslant i \leqslant r$. It follows that $s=0$ if and only if $x_{i, j} / x_{i, 0}=y_{i, j} / y_{i, 0}$ for all $1 \leqslant i \leqslant r$ and $1 \leqslant j \leqslant n_{i}$. Hence, the global section $s$ vanishes precisely on the diagonal $\mathbb{P}^{\boldsymbol{n}} \hookrightarrow \mathbb{P}^{\boldsymbol{n}} \times \mathbb{P}^{\boldsymbol{n}}$.

The Koszul complex associated with $s$ is the required locally free resolution of the diagonal because $\mathbb{P}^{\boldsymbol{n}}$ is smooth and the codimension of the diagonal equals the rank of the vector bundle $\bigoplus_{i=1}^{r} \mathcal{O}_{\mathbb{P} n}\left(\boldsymbol{e}_{i}\right) \otimes \mathcal{T}_{\mathbb{P} \boldsymbol{n}}^{\boldsymbol{e}_{i}}\left(-\boldsymbol{e}_{i}\right)$; see [Laz04, Section B.2]. Since $\Omega_{\mathbb{P} \boldsymbol{n}}^{\boldsymbol{e}_{i}}=\mathcal{H}_{\mathrm{om}} \mathrm{O}_{\mathbb{P} \boldsymbol{n}}\left(\mathcal{T}_{\mathbb{P} \boldsymbol{n}}^{\boldsymbol{e}_{i}}, \mathcal{O}_{\mathbb{P} \boldsymbol{n}}\right)$, we have

$$
\bigwedge^{k}\left(\mathcal{O}_{\mathbb{P} n}\left(-e_{i}\right) \otimes \Omega_{\mathbb{P} n}^{e_{i}}\left(\boldsymbol{e}_{i}\right)\right)=\bigoplus_{\substack{0 \leqslant \boldsymbol{u} \leqslant \boldsymbol{n} \\|\boldsymbol{u}|=k}} \mathcal{O}_{\mathbb{P} \boldsymbol{n}}(-\boldsymbol{u}) \otimes \Omega_{\mathbb{P} \boldsymbol{n}}^{\boldsymbol{u}}(\boldsymbol{u})
$$

for $0 \leqslant k \leqslant|\boldsymbol{n}|$.

Proof of Proposition 1.2. Let $\pi_{1}$ and $\pi_{2}$ be the projections of $\mathbb{P}^{\boldsymbol{n}} \times \mathbb{P}^{\boldsymbol{n}}$ onto the first and second factors, respectively. For any $\boldsymbol{u} \in \mathbb{Z}^{r}$, the Fujita vanishing theorem [Fuj83, Theorem 1] implies that $\Omega_{\mathbb{P} \boldsymbol{n}}^{\boldsymbol{u}}(\boldsymbol{u}+\boldsymbol{d}) \otimes \widetilde{M}$ has no higher cohomology for any sufficiently positive $\boldsymbol{d} \in \mathbb{Z}^{r}$. Let $\mathcal{K}$ be the locally free resolution of the diagonal $\mathbb{P}^{\boldsymbol{n}} \hookrightarrow \mathbb{P}^{\boldsymbol{n}} \times \mathbb{P}^{\boldsymbol{n}}$ described in Lemma 2.1. Both hypercohomology spectral sequences, namely

$$
{ }^{\prime} \mathrm{E}_{2}^{p, q}:=\mathrm{H}^{p}\left(\mathbf{R}^{q} \pi_{1 *}\left(\pi_{2}^{*} \widetilde{M}(\boldsymbol{d}) \otimes_{\mathcal{O}_{\mathbb{P}} \boldsymbol{n}_{\times \mathbb{P}} \boldsymbol{n}} \mathcal{K}\right)\right) \quad \text { and } \quad " \quad \mathrm{E}_{2}^{p, q}:=\mathbf{R}^{p} \pi_{1 *} \mathrm{H}^{q}\left(\pi_{2}^{*} \widetilde{M}(\boldsymbol{d}) \otimes_{\mathcal{O}_{\mathbb{P}} \boldsymbol{n}_{\times \mathbb{P}} \boldsymbol{n}} \mathcal{K}\right),
$$

converge to $\mathbf{R}^{p+q} \pi_{1_{*}}\left(\pi_{2}^{*} \widetilde{M}(\boldsymbol{d}) \otimes_{\mathcal{O}_{\mathbb{P}} \boldsymbol{n}_{\times \mathbb{P}} \boldsymbol{n}} \mathcal{K}\right)$; see [GD61, Section 12.4]. Since $\mathcal{K}$ is a locally free resolution of the diagonal, it follows that ${ }^{\prime \prime} \mathrm{E}_{2}^{0,0} \cong \widetilde{M}(\boldsymbol{d})$ and ${ }^{\prime \prime} \mathrm{E}_{2}^{p, q}=0$ when either $p \neq 0$ or $q \neq 0$; compare with [Huy06, Proposition 8.28]. Hence, we conclude that

$$
\mathbf{R}^{p+q} \pi_{1 *}\left(\pi_{2}^{*} \widetilde{M}(\boldsymbol{d}) \otimes_{\mathcal{O}_{\mathbb{P}} \boldsymbol{n}_{\times \mathbb{P}} \boldsymbol{n}} \mathcal{K}\right) \cong \begin{cases}\widetilde{M}(\boldsymbol{d}) & \text { if } p=0=q, \\ 0 & \text { otherwise }\end{cases}
$$

On the other hand, the first page of the other hypercohomology spectral sequence is

$$
\begin{aligned}
& { }^{\prime} \mathrm{E}_{1}^{p, q}=\mathbf{R}^{q} \pi_{1 *}\left(\pi_{2}^{*} \widetilde{M}(\boldsymbol{d}) \otimes_{\mathcal{O}_{\mathbb{P}} \boldsymbol{n}_{\times \mathbb{P}} \boldsymbol{K}} \mathcal{K}_{-p}\right)=\mathbf{R}^{q} \pi_{1 *}\left(\underset{\substack{\mathbf{0} \leqslant \boldsymbol{u} \leqslant \boldsymbol{n} \\
|\boldsymbol{u}|=-p}}{\bigoplus} \mathcal{O}_{\mathbb{P} \boldsymbol{n}}(-\boldsymbol{u}) \otimes\left(\Omega_{\mathbb{P} \boldsymbol{n}}^{\boldsymbol{u}} \otimes \widetilde{M}(\boldsymbol{u}+\boldsymbol{d})\right)\right) \\
& =\bigoplus_{\substack{0 \leqslant \boldsymbol{u} \leqslant \boldsymbol{n} \\
|\boldsymbol{u}|=-p}} \mathcal{O}_{\mathbb{P} \boldsymbol{n}}(-\boldsymbol{u}) \otimes_{\mathbb{k}} H^{q}\left(\mathbb{P}^{\boldsymbol{n}}, \Omega_{\mathbb{P} \boldsymbol{n}}^{\boldsymbol{u}} \otimes \widetilde{M}(\boldsymbol{u}+\boldsymbol{d})\right) .
\end{aligned}
$$

Our positivity assumption on $\boldsymbol{d}$ implies that $H^{q}\left(\mathbb{P}^{\boldsymbol{n}}, \Omega_{\mathbb{P} \boldsymbol{n}}^{\boldsymbol{u}} \otimes \widetilde{M}(\boldsymbol{u}+\boldsymbol{d})\right)=0$ for all $q>0$, so ${ }^{\prime} \mathrm{E}_{1}^{p, q}$ is concentrated in a single row. Applying the functor $\mathcal{F} \mapsto \bigoplus_{\boldsymbol{v} \in \mathbb{N}^{r}} H^{0}\left(\mathbb{P}^{\boldsymbol{n}}, \mathcal{F}(\boldsymbol{v})\right)$, we obtain a virtual resolution of $M$ in which the $i$ th module is

$$
\bigoplus_{\substack{0 \leqslant \boldsymbol{u} \leqslant \boldsymbol{n} \\|\boldsymbol{u}|=i}} S(-\boldsymbol{u}) \otimes_{\mathbb{k}} H^{q}\left(\mathbb{P}^{\boldsymbol{n}}, \Omega_{\mathbb{P}^{\boldsymbol{n}}}^{\boldsymbol{u}} \otimes \widetilde{M}(\boldsymbol{u}+\boldsymbol{d})\right) .
$$

Remark 2.2. By scrutinizing the linear free resolutions of well-chosen truncated twisted modules, Corollary 2.14 in [EES15] also establishes the existence of short virtual resolutions on $\mathbb{P}^{n}$. Although the proof of Proposition 1.2 and [EES15, Proposition 2.7] use somewhat different notions of a "sufficiently positive" degree $\boldsymbol{d} \in \mathbb{Z}^{r}$, both are quite similar to Castelnuovo-Mumford regularity. 


\section{Berkesch, D. Erman and G.G. Smith}

The next examples demonstrate why we want more than just these short virtual resolutions arising from the proof of Proposition 1.2.

Example 2.3. Consider the hyperelliptic curve $C \subset \mathbb{P}^{1} \times \mathbb{P}^{2}$ from Example 1.4. Using $\boldsymbol{d}:=(2,2)$ in the construction from the proof of Proposition 1.2 yields a virtual resolution of the form

$$
S(-2,-2)^{17} \longleftarrow \underset{S(-3,-2)^{15}}{\oplus(-2,-3)^{26}} \longleftarrow \underset{S(-3,-3)^{22}}{\oplus} \longleftarrow S(-3,-4)^{7} \longleftarrow 0 .
$$

Compared to the virtual resolution in (1.2), the length of this complex is longer, the rank of the free modules is higher, and the degrees of the generators are larger.

Example 2.4. If $X$ is the union of $m$ distinct points on $\mathbb{P}^{1} \times \mathbb{P}^{1}$, then for any sufficiently positive $\boldsymbol{d}=\left(d_{1}, d_{2}\right)$, the construction in the proof of Proposition 1.2 yields a virtual resolution of the form

$$
S\left(-d_{1},-d_{2}\right)^{m} \longleftarrow \begin{gathered}
S\left(-d_{1}-1,-d_{2}\right)^{m} \\
S\left(-d_{1},-d_{2}-1\right)^{m}
\end{gathered} \longleftarrow S\left(-d_{1}-1,-d_{2}-1\right)^{m} \longleftarrow 0 .
$$

Unlike the minimal free resolution, the Betti table of this free complex is independent of the geometry of the points, so even short virtual resolutions can obscure the geometric information.

As a counterpoint to Proposition 1.2, we provide a lower bound on the length of a virtual resolution. Extending the well-known result for projective space, we show that the codimension of any associated prime of $M$ gives a lower bound on the length of any virtual resolution of $M$.

Proposition 2.5. Let $M$ be a finitely generated $\mathbb{Z}^{r}$-graded $S$-module. Let $Q$ be an associated prime of $M$ that does not contain the irrelevant ideal $B$, and let

$$
F:=\left[F_{0} \longleftarrow F_{1} \longleftarrow \cdots \longleftarrow F_{p} \longleftarrow 0\right]
$$

be a virtual resolution of $M$. These hypotheses yield the following:

(i) We have $\operatorname{codim} Q \leqslant p$.

(ii) If $Q$ is the prime ideal for a closed point of $\mathbb{P}^{\boldsymbol{n}}$, then we have $p \geqslant|\boldsymbol{n}|$.

(iii) If $p \leqslant \min \left\{n_{i}+1: 1 \leqslant i \leqslant r\right\}$, then $F$ is a free resolution of $\mathrm{H}_{0}(F)$.

Proof. When we localize at the prime ideal $Q$, the resolution $F$ becomes a free $S_{Q}$-resolution of $M_{Q}$. Part (i) then follows from the fact that, over the local ring $S_{Q}$, the projective dimension of a module is always greater than or equal to the codimension of a module; see [Eis95, Proposition 18.2]. Part (ii) is immediate, as $\operatorname{codim} Q=|\boldsymbol{n}|$ if $Q$ is the prime ideal for a closed point of $\mathbb{P}^{\boldsymbol{n}}$.

For part (iii), assume to the contrary that $F$ is not a free resolution of $\mathrm{H}_{0}(F)$. It follows that $\mathrm{H}_{j}(F) \neq 0$ for some $j>0$; choose the maximal such $j$. Since $F$ is a virtual resolution of $M$, the module $\mathrm{H}_{j}(F)$ must be supported on the irrelevant ideal $B$. Let $P_{i}:=\left\langle x_{i, 0}, x_{i, 1}, \ldots, x_{i, n_{i}}\right\rangle$ be the component of the irrelevant ideal $B$ corresponding to the factor $\mathbb{P}^{n_{i}}$; there is an index $i$ such that $\left(\mathrm{H}_{j}(F)\right)_{P_{i}} \neq 0$. Localizing at $P_{i}$ yields a complex $F_{P_{i}}$ of the form

$$
\cdots \longleftarrow\left(F_{j-1}\right)_{P_{i}} \longleftarrow\left(F_{j}\right)_{P_{i}} \longleftarrow\left(F_{j+1}\right)_{P_{i}} \longleftarrow \cdots \longleftarrow\left(F_{p}\right)_{P_{i}} \longleftarrow 0
$$

where the homology $\mathrm{H}_{j}\left(F_{P_{i}}\right)$ is supported on the maximal ideal of the local ring $S_{P_{i}}$. We deduce that $p>p-j \geqslant \operatorname{codim} P_{i}=n_{i}+1$ from the Peskine-Szpiro acyclicity lemma; see [Eis95, Lemma 20.11]. However, this contradicts our assumption that $p \leqslant \min \left\{n_{i}+1: 1 \leqslant i \leqslant r\right\}$. Therefore, we conclude that the complex $F$ is a free resolution of $\mathrm{H}_{0}(F)$. 


\section{VIRTUAL RESOLUTIONS}

The following simple corollary is useful in applications such as Theorem 1.6.

Corollary 2.6. Let $I$ be a $B$-saturated $S$-ideal, and let $F=\left[F_{0} \longleftarrow F_{1} \longleftarrow \cdots \longleftarrow F_{p} \longleftarrow 0\right]$ be a virtual resolution of $S / I$. If $F_{0}=S$ and $p<\min \left\{n_{i}+1: 1 \leqslant i \leqslant r\right\}$, then the complex $F$ is a free resolution of $S / I$.

Proof. By part (iii) of Proposition 2.5, the complex $F$ is a free resolution of $\mathrm{H}_{0}(F)$. The hypothesis $F_{0}=S$ implies that $\mathrm{H}_{0}(F)=S / J$ for some ideal $J$. Since $I$ is $B$-saturated and $F$ is a virtual resolution of $S / I$, we deduce that $I$ equals the $B$-saturation of $J$. If we had $I \neq J$, then it would follow that $S / J$ has an associated prime $Q$ that contains the irrelevant ideal $B$. However, the codimension of $Q$ is at least $\min \left\{n_{i}+1: 1 \leqslant i \leqslant r\right\}$. As $F$ is a free resolution of $S / J$, this would yield $p \geqslant \min \left\{n_{i}+1: 1 \leqslant i \leqslant r\right\}$ and therefore a contradiction; see [Eis95, Proposition 18.2].

Just like in projective space, one can find subvarieties of codimension $c$ which do not admit a virtual resolution of length $c$.

Example 2.7. Working in $\mathbb{P}^{2} \times \mathbb{P}^{2}$, consider the $B$-saturated $S$-ideal $J:=\left\langle x_{1,0}, x_{1,1}\right\rangle \cap\left\langle x_{2,0}, x_{2,1}\right\rangle$. The minimal free resolution of $S / J$ has the form

$$
S \longleftarrow S(-1,-1)^{4} \longleftarrow \underset{S(-1,-2)^{2}}{\oplus(-2,-1)^{2}} \longleftarrow S(-2,-2) \longleftarrow 0 .
$$

Although the codimension of every associated prime of $J$ is 2 , there is no virtual resolution of $S / J$ of length 2. If we had such a free complex $F=\left[F_{0} \longleftarrow F_{1} \longleftarrow F_{2} \longleftarrow 0\right]$, then Corollary 2.6 would imply that $F$ is a minimal free resolution of $S / J$, which would give a contradiction.

Remark 2.8. Proposition 5.1 analyzes when a subscheme has a virtual resolution of its structure sheaf whose length equals its codimension - a special case of equality in part (i) of Proposition 2.5.

We next refine our results on short virtual resolutions by developing effective degree bounds. Following [MS04, Definition 1.1], a finitely generated $\mathbb{Z}^{r}$-graded $B$-saturated $S$-module $M$ is $\boldsymbol{m}$-regular, for some $\boldsymbol{m} \in \mathbb{Z}^{r}$, if $H_{B}^{i}(M)_{\boldsymbol{p}}=0$ for all $i \geqslant 1$ and all $\boldsymbol{p} \in \bigcup\left(\boldsymbol{m}-\boldsymbol{q}+\mathbb{N}^{r}\right)$, where the union is over all $\boldsymbol{q} \in \mathbb{N}^{r}$ such that $|\boldsymbol{q}|=i-1$. The (multigraded Castelnuovo-Mumford) regularity of $M$ is $\operatorname{reg} M:=\left\{\boldsymbol{p} \in \mathbb{Z}^{r}: M\right.$ is $\boldsymbol{p}$-regular $\}$. Let $\Delta_{i} \subset \mathbb{Z}^{r}$ denote the set of twists of the summands in the $i$ th step of the minimal free resolution of the irrelevant ideal $B$.

TheOREM 2.9. For a finitely generated $\mathbb{Z}^{r}$-graded $B$-saturated $S$-module $M$, we have $\boldsymbol{d} \in \operatorname{reg} M$ if and only if the module $M(\boldsymbol{d})$ has a virtual resolution $F_{0} \longleftarrow F_{1} \longleftarrow \cdots \longleftarrow F_{|\boldsymbol{n}|} \longleftarrow 0$ such that for all $0 \leqslant i \leqslant|\boldsymbol{n}|$, the degree of each generator of $F_{i}$ belongs to $\Delta_{i}+\mathbb{N}^{r}$ and its Hilbert polynomial and Hilbert function agree on $\mathbb{N}^{r}$.

When $r=1$, we have $\Delta_{i}=\{-i\}$, and this theorem specializes to the existence of linear resolutions on projective space; see [Laz04, Proposition 1.8.8]. Since the minimal free resolution of $S / B$ is a cellular resolution described explicitly by [BS98, Corollary 2.13], it follows that $\Delta_{0}:=\{\mathbf{0}\}$ and that for $i \geqslant 1$, we have $\Delta_{i}:=\left\{-\boldsymbol{a} \in \mathbb{Z}^{r}: \mathbf{0} \leqslant \boldsymbol{a}-\mathbf{1} \leqslant \boldsymbol{n}\right.$ and $\left.|\boldsymbol{a}|=r+i-1\right\}$. We first illustrate Theorem 2.9 in the case of a hypersurface.

Example 2.10. Given a homogeneous polynomial $f \in S$ of degree $\boldsymbol{d}$, the regularity of $S /\langle f\rangle$ has a unique minimal element $\boldsymbol{e}$, where $e_{j}:=\max \left\{0, d_{j}-1\right\}$. As a consequence, it follows that $\mathbf{0} \in \operatorname{reg}(S /\langle f\rangle)$ if and only if $d_{j} \leqslant 1$ for all $j$.

Before proving Theorem 2.9, we need two technical lemmas. 


\section{Berkesch, D. Erman and G.G. Smith}

Lemma 2.11. For $0 \leqslant i \leqslant|\boldsymbol{n}|, \boldsymbol{b} \in \Delta_{i}+\mathbb{N}^{r}$, and $\boldsymbol{a} \in \mathbb{N}^{r} \backslash\{\mathbf{0}\}$, we have $H^{|\boldsymbol{a}|+i}\left(\mathbb{P}^{\boldsymbol{n}}, \mathcal{O}_{\mathbb{P} \boldsymbol{n}}(\boldsymbol{b}-\boldsymbol{a})\right)=0$.

Proof. We induct on $r$. For the base case $r=1$, we have nonzero higher cohomology for the given line bundle on $\mathbb{P}^{n_{1}}$ only if $|\boldsymbol{a}|+i=a_{1}+i=n_{1}$. Since $\boldsymbol{b} \in \Delta_{i}+\mathbb{N}=\{-i\}+\mathbb{N}$, or $b_{1} \geqslant-i$, we have $b_{1}-a_{1} \geqslant-i-\left(n_{1}-i\right)=-n_{1}>-n_{1}-1$, so $\mathcal{O}_{\mathbb{P} n}\left(b_{1}-a_{1}\right)$ has no higher cohomology.

For the induction step, we first consider the case where at least one entry of $\boldsymbol{b}-\boldsymbol{a}$ is nonnegative, and we assume for a contradiction that $H^{|\boldsymbol{a}|+i}\left(\mathbb{P}^{\boldsymbol{n}}, \mathcal{O}_{\mathbb{P} \boldsymbol{n}}(\boldsymbol{b}-\boldsymbol{a})\right) \neq 0$. Since $|\boldsymbol{a}|+i>0$, we may also assume, by reordering the factors, that the first entry of $\boldsymbol{b}-\boldsymbol{a}$ is strictly negative and the last entry is nonnegative. We write $\boldsymbol{b}-\boldsymbol{a}=\left(\boldsymbol{b}^{\prime}-\boldsymbol{a}^{\prime}, b_{r}-a_{r}\right)$ and $\boldsymbol{n}=\left(\boldsymbol{n}^{\prime}, n_{r}\right)$ in $\mathbb{Z}^{r-1} \oplus \mathbb{Z}$. Since $H^{|\boldsymbol{a}|+i}\left(\mathbb{P}^{\boldsymbol{n}}, \mathcal{O}_{\mathbb{P} \boldsymbol{n}}(\boldsymbol{b}-\boldsymbol{a})\right) \neq 0$, the Künneth formula implies that $H^{|\boldsymbol{a}|+i}\left(\mathbb{P}^{\boldsymbol{n}^{\prime}}, \mathcal{O}_{\mathbb{P} \boldsymbol{n}^{\prime}}\left(\boldsymbol{b}^{\prime}-\boldsymbol{a}^{\prime}\right)\right) \neq 0$. Decreasing the first entry of $\boldsymbol{b}^{\prime}-\boldsymbol{a}^{\prime}$ will not alter this nonvanishing. Setting $\boldsymbol{a}^{\prime \prime}:=\boldsymbol{a}^{\prime}+\left(a_{r}, 0,0, \ldots, 0\right)$, we obtain $\boldsymbol{a}^{\prime \prime} \in \mathbb{N}^{r-1} \backslash\{\mathbf{0}\},\left|\boldsymbol{a}^{\prime \prime}\right|=\left|\boldsymbol{a}^{\prime}\right|+a_{r}=|\boldsymbol{a}|$, and $H^{\left|\boldsymbol{a}^{\prime \prime}\right|+i}\left(\mathbb{P}^{\boldsymbol{n}^{\prime}}, \mathcal{O}_{\mathbb{p} \boldsymbol{n}^{\prime}}\left(\boldsymbol{b}^{\prime}-\boldsymbol{a}^{\prime \prime}\right)\right) \neq 0$, which contradicts the induction hypothesis.

It remains to consider the case where all entries of $\boldsymbol{b}-\boldsymbol{a}$ are strictly negative. Hence, we can assume $|\boldsymbol{a}|+i=|\boldsymbol{n}|$. The hypothesis $\boldsymbol{b} \in \Delta_{i}+\mathbb{N}^{r}$ implies that $|\boldsymbol{b}| \geqslant-r-i+1$. Combining these yields $|\boldsymbol{b}-\boldsymbol{a}|=\sum_{i} b_{i}-a_{i}=|\boldsymbol{b}|-|\boldsymbol{a}| \geqslant(-r-i+1)-(|\boldsymbol{n}|-i)=-|\boldsymbol{n}|-r+1$. But the most positive line bundle with top-dimensional cohomology is the canonical bundle, and this inequality shows that $\mathcal{O}_{\mathbb{P} n}(\boldsymbol{b}-\boldsymbol{a})$ cannot have top-dimensional cohomology.

Remark 2.12. Proposition 5.7 develops a related vanishing result for derived pushforwards.

Lemma 2.13. Let $\mathcal{F}$ be a $\mathbf{0}$-regular $\mathcal{O}_{\mathbb{P} \boldsymbol{n}}$-module, and let $\mathbf{0} \leqslant \boldsymbol{a} \leqslant \boldsymbol{n}$. If $H^{p}\left(\mathbb{P}^{\boldsymbol{n}}, \mathcal{F} \otimes \Omega^{\boldsymbol{a}}(\boldsymbol{a})\right) \neq 0$, then we have $-\boldsymbol{a} \in \Delta_{|\boldsymbol{a}|-p}+\mathbb{N}^{r}$.

Proof. If $\boldsymbol{a}=\mathbf{0}$, then we have $\Omega^{\boldsymbol{a}}(\boldsymbol{a})=\mathcal{O}_{\mathbb{P} \boldsymbol{n}}$, and the statement follows immediately from the $\mathbf{0}$-regularity of $\mathcal{F}$. Thus, we assume $\boldsymbol{a} \neq \mathbf{0}$. After possibly reordering the factors of $\mathbb{P}^{\boldsymbol{n}}$, we may write $\boldsymbol{a}=\left(\boldsymbol{a}^{\prime}, \mathbf{0}\right) \in \mathbb{Z}^{r^{\prime}} \oplus \mathbb{Z}^{r-r^{\prime}}$, where every entry of $\boldsymbol{a}^{\prime}$ is strictly positive. For any $k \geqslant 1$, we have $\left(-\boldsymbol{a}^{\prime}, \mathbf{0}\right) \in \Delta_{k}+\mathbb{N}^{r} \Longleftrightarrow\left(-\boldsymbol{a}^{\prime},-\mathbf{1}\right) \in \Delta_{k}+\mathbb{N}^{r} \Longleftrightarrow\left|\boldsymbol{a}^{\prime}\right|+\left(r-r^{\prime}\right) \leqslant k+r-1$. Setting $k=|\boldsymbol{a}|-p=\left|\boldsymbol{a}^{\prime}\right|-p$ establishes that $-\boldsymbol{a}=\left(-\boldsymbol{a}^{\prime}, \mathbf{0}\right) \in \Delta_{|\boldsymbol{a}|-p}+\mathbb{N}^{r}$ is equivalent to $p<r^{\prime}$.

We next use truncated Koszul complexes to build a locally free resolution of $\Omega_{\mathbb{P} \boldsymbol{n}}^{\boldsymbol{a}}(\boldsymbol{a})$. For $j>r^{\prime}$, we have $a_{j}=0$ and $\Omega_{\mathbb{P}^{n_{j}}}^{a_{j}}\left(a_{j}\right) \cong \mathcal{O}_{\mathbb{P}^{n_{j}}}$. For $1 \leqslant j \leqslant r^{\prime}$, the truncated Koszul complex twisted by $\mathcal{O}_{\mathbb{P}^{n_{j}}}\left(a_{j}\right)$, namely

$$
\mathcal{O}_{\mathbb{P}^{n_{j}}}(-1)^{\left(\begin{array}{c}
n_{j}+1 \\
a_{j}+1
\end{array}\right)} \longleftarrow \mathcal{O}_{\mathbb{P}^{n_{j}}}(-2)^{\left(\begin{array}{c}
n_{j}+1 \\
a_{j}+2
\end{array}\right)} \longleftarrow \cdots \longleftarrow \mathcal{O}_{\mathbb{P}^{n_{j}}}\left(-n_{j}-1+a_{j}\right)^{\left(\begin{array}{c}
n_{j}+1 \\
n_{j}+1
\end{array}\right)} \longleftarrow 0,
$$

resolves $\Omega_{\mathbb{P}^{n_{j}}}^{a_{j}}\left(a_{j}\right)$. Taking external tensor products gives a locally free resolution $\mathcal{G}$ of $\Omega_{\mathbb{P}^{\boldsymbol{n}}}^{\boldsymbol{a}}(\boldsymbol{a})$. Any summand $\mathcal{O}_{\mathbb{P} \boldsymbol{n}}(\boldsymbol{c})$ in $\mathcal{G}_{i}$ has the form $\boldsymbol{c}=\left(\boldsymbol{c}^{\prime}, \mathbf{0}\right) \in \mathbb{Z}^{r^{\prime}} \oplus \mathbb{Z}^{r-r^{\prime}}$, where $\left|\boldsymbol{c}^{\prime}\right|=-r^{\prime}-i$. Tensoring the locally free resolution $\mathcal{G}$ with $\mathcal{F}$ gives a resolution of $\mathcal{F} \otimes \Omega_{\mathbb{P} \boldsymbol{n}}^{a}(\boldsymbol{a})$.

Since $H^{p}\left(\mathcal{F} \otimes \Omega_{\mathbb{p} \boldsymbol{n}}^{\boldsymbol{a}}(\boldsymbol{a})\right) \neq 0$, breaking the resolution $\mathcal{F} \otimes \mathcal{G}$ into short exact sequences implies that for some index $i$, we have $H^{p+i}\left(\mathcal{F} \otimes \mathcal{G}_{i}\right) \neq 0$. Hence, there exists a $\boldsymbol{c}=\left(\boldsymbol{c}^{\prime}, \mathbf{0}\right)$ with $\left|\boldsymbol{c}^{\prime}\right|=-r^{\prime}-i$ such that $H^{p+i}\left(\mathbb{P}^{\boldsymbol{n}}, \mathcal{F}(\boldsymbol{c})\right) \neq 0$. Since $\mathcal{F}$ is $\mathbf{0}$-regular, we have $|\boldsymbol{c}|=\left|\boldsymbol{c}^{\prime}\right|<-(p+i)$. Therefore, we conclude that $p<-\left|\boldsymbol{c}^{\prime}\right|-i=\left(r^{\prime}+i\right)-i=r^{\prime}$ and $\boldsymbol{a} \in \Delta_{|\boldsymbol{a}|-p}+\mathbb{N}^{r}$.

Proof of Theorem 2.9. Assume that $M(\boldsymbol{d})$ has a virtual resolution $F$ of the specified form and that its Hilbert polynomial and Hilbert function agree on $\mathbb{N}^{r}$. Since $M$ is $B$-saturated, it suffices to show that $H^{|\boldsymbol{a}|}\left(\mathbb{P}^{\boldsymbol{n}}, \widetilde{M}(\boldsymbol{d}-\boldsymbol{a})\right)=0$ for all $\boldsymbol{a} \in \mathbb{N}^{r}-\{\mathbf{0}\}$. By splitting up $F$ into short exact sequences, it suffices to show that $H^{|\boldsymbol{a}|+i}\left(\mathbb{P}^{\boldsymbol{n}}, \widetilde{F}_{i}(-\boldsymbol{a})\right)=0$ for all $\boldsymbol{a} \in \mathbb{N}^{r} \backslash\{\mathbf{0}\}$. This is the content of Lemma 2.11. 


\section{VIRTUAL RESOLUTIONS}

For the converse, let $\mathcal{K}$ denote the locally free resolution of the diagonal $\mathbb{P}^{\boldsymbol{n}} \hookrightarrow \mathbb{P}^{\boldsymbol{n}} \times \mathbb{P}^{\boldsymbol{n}}$ described in Lemma 2.1. Let $\pi_{1}$ and $\pi_{2}$ be the projections onto the first and second factors of $\mathbb{P}^{n} \times \mathbb{P}^{n}$, respectively. The sheaf $\widetilde{M}(\boldsymbol{d})$ is quasi-isomorphic to the complex $\mathcal{F}=\mathbf{R} \pi_{1 *}\left(\pi_{2}^{*} \widetilde{M}(\boldsymbol{d}) \otimes \mathcal{K}\right)$, where

$$
\mathcal{F}_{j}=\bigoplus_{|\boldsymbol{a}|-p=j} H^{p}\left(\mathbb{P}^{\boldsymbol{n}}, \widetilde{M}(\boldsymbol{d}) \otimes \Omega_{\mathbb{P} \boldsymbol{n}}^{\boldsymbol{a}}(\boldsymbol{a})\right) \otimes \mathcal{O}_{\mathbb{P} \boldsymbol{n}}(-\boldsymbol{a}) .
$$

Lemma 2.13 says that $H^{p}\left(\mathbb{P}^{n}, \widetilde{M}(\boldsymbol{d}) \otimes \Omega_{\mathbb{P} \boldsymbol{n}}^{\boldsymbol{a}}(\boldsymbol{a})\right) \neq 0$ only if $-\boldsymbol{a} \in \Delta_{|\boldsymbol{a}|-p}+\mathbb{N}^{r}=\Delta_{j}+\mathbb{N}^{r}$. Since each $\mathcal{F}_{j}$ is a sum of line bundles, the corresponding $S$-module $F_{j}$ is free. It follows that the complex $F:=\left[F_{0} \longleftarrow F_{1} \longleftarrow \cdots \longleftarrow F_{|\boldsymbol{n}|} \longleftarrow 0\right]$ is a virtual resolution of $M(\boldsymbol{d})$ with the desired form. Finally, the module $M$ is $B$-saturated so $H_{B}^{0}(M)=0$, and the hypothesis $\boldsymbol{d} \in \operatorname{reg} M$ implies that $H_{B}^{1}(M(\boldsymbol{d}))_{\boldsymbol{p}}=0$ for all $\boldsymbol{p} \in \mathbb{N}^{r}$, so the Hilbert polynomial and Hilbert function of $M(\boldsymbol{d})$ agree on $\mathbb{N}^{r}$.

\section{Simpler virtual resolutions}

We describe, in this section, an effective method for producing interesting virtual resolutions of a given $S$-module. Unlike in the previous section, the free complex is ordinarily not linear or acyclic. Our construction depends on a $B$-saturated module $M$ as well as an element $\boldsymbol{d} \in \operatorname{reg} M$. Although Theorem 3.1 defines the corresponding virtual resolution as a subcomplex of a minimal free resolution of $M$, Algorithm 3.4 shows that the subcomplex can be assembled without first computing the entire minimal free resolution.

Theorem 3.1. For a finitely generated $\mathbb{Z}^{r}$-graded $S$-module $M$, consider a minimal free resolution $F$ of $M$. For a degree $\boldsymbol{d} \in \mathbb{Z}^{r}$ and each $i$, let $G_{i}$ be the direct sum of all free summands of $F_{i}$ whose generator is in degree at most $\boldsymbol{d}+\boldsymbol{n}$, and let $\varphi_{i}$ be the restriction of the ith differential of $F$ to $G_{i}$.

(i) For all $i$, we have $\varphi_{i}\left(G_{i}\right) \subseteq G_{i-1}$ and $\varphi_{i} \circ \varphi_{i+1}=0$, so $G$ forms a free complex.

(ii) Up to isomorphism, $G$ depends only on $M$ and $\boldsymbol{d}$.

(iii) If $M$ is $B$-saturated and $\boldsymbol{d} \in \operatorname{reg} M$, then the complex $G$ is a virtual resolution of $M$.

When $M$ is $B$-saturated and $\boldsymbol{d} \in \operatorname{reg} M$, the complex $G$ is the virtual resolution of the pair $(M, \boldsymbol{d})$.

Proof of Theorem 1.3. This theorem is simply a restatement of part (iii) of Theorem 3.1.

To illustrate the basic idea behind the proof of Theorem 3.1, we revisit our first example.

Example 3.2. Let $C$ be the hyperelliptic curve in $\mathbb{P}^{1} \times \mathbb{P}^{2}$ defined by the ideal $I$ in Example 1.4. The free complex in (1.2) is the virtual resolution of the pair $(S / I,(4,2))$, and it is a subcomplex of the minimal free resolution (1.1) of $S / I$. The corresponding quotient complex $E$ is

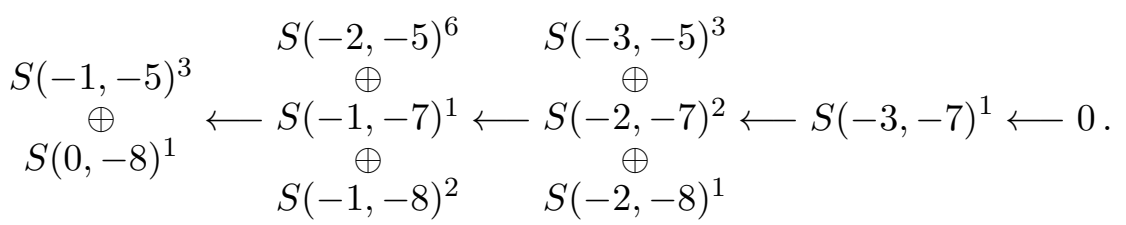

Restricting attention to the terms of degree $(*,-8)$, we have

$$
S(0,-8)^{1} \longleftarrow S(-1,-8)^{2} \longleftarrow S(-2,-8)^{1} \longleftarrow 0,
$$




\section{Berkesch, D. Erman and G.G. Smith}

which looks like a twist of the Koszul complex on $x_{1,0}$ and $x_{1,1}$. In fact, the $(*,-8),(*,-7)$, and $(*,-5)$ strands each appear to have homology supported on the irrelevant ideal. This suggests that the complex $\widetilde{E}$ is quasi-isomorphic to zero, and that is what we show in the proof of Theorem 3.1.

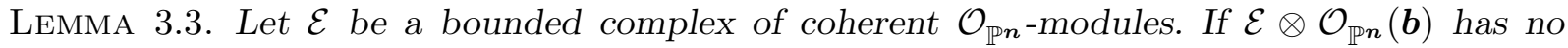
hypercohomology for all $\mathbf{0} \leqslant \boldsymbol{b} \leqslant \boldsymbol{n}$, then $\mathcal{E}$ is quasi-isomorphic to 0 .

Proof. By [EES15, Theorem 1.1], any bounded complex of coherent $\mathcal{O}_{\mathbb{P} n}$-modules is quasiisomorphic to a Beilinson monad whose terms involve the hypercohomology evaluated at the line bundles of the form $\mathbf{0} \leqslant \boldsymbol{b} \leqslant \boldsymbol{n}$. The hypothesis on vanishing hypercohomology ensures that this Beilinson monad of $\mathcal{E}$ is the 0 monad, and hence $\mathcal{E}$ is quasi-isomorphic to 0. While [EES15, Theorem 1.1] is stated for a sheaf, the authors remark in equation (1) on page 8 that a similar statement holds for bounded complexes of coherent sheaves.

Proof of Theorem 3.1. For part (i), write $F_{i}=G_{i} \oplus E_{i}$ for each $i$. Each generating degree $\boldsymbol{e}$ of $E_{i}$ satisfies $\boldsymbol{e} \mathbb{d}+\boldsymbol{n}$. It follows that, for degree reasons, there are no nonzero maps from $G_{i}$ to $E_{i-1}$. The $i$ th differential $\partial_{i}: F_{i} \rightarrow F_{i-1}$ has a block decomposition

$$
\begin{aligned}
& G_{i} \quad E_{i} \\
& \partial_{i}=\begin{array}{c}
G_{i-1} \\
E_{i-1}
\end{array}\left[\begin{array}{cc}
\varphi_{i} & * \\
0 & *
\end{array}\right],
\end{aligned}
$$

so $\varphi_{i}\left(G_{i}\right) \subseteq G_{i-1}$ and $\partial_{i} \circ \partial_{i+1}=0$ implies that $\varphi_{i} \circ \varphi_{i+1}=0$. As $G$ depends only on $F$ and $\boldsymbol{d}$, part (ii) follows from the fact that the minimal free resolution of $M$ is unique up to isomorphism. For part (iii), we may replace $M$ with $M(\boldsymbol{d})$ and $\boldsymbol{d}$ with $\mathbf{0}$. Let $G$ be the virtual resolution of the pair $(M, \mathbf{0})$, and consider the short exact sequence of complexes $0 \rightarrow G \rightarrow F \rightarrow E \rightarrow 0$. It suffices to show that the complex $\widetilde{E}$ of sheaves is quasi-isomorphic to zero.

Fix some $\boldsymbol{b}$, where $\mathbf{0} \leqslant \boldsymbol{b} \leqslant \boldsymbol{n}$. If $\boldsymbol{a} \nless \boldsymbol{n}$, then the line bundle $\mathcal{O}_{\mathbb{P} \boldsymbol{n}}(-\boldsymbol{a}+\boldsymbol{b})$ has no global sections. It follows that each summand of $\widetilde{F}(\boldsymbol{b})$ with global sections belongs to $\widetilde{G}(\boldsymbol{b})$. If $H^{i}\left(\mathbb{P}^{n}, \widetilde{F}(\boldsymbol{b})\right)$ is the complex obtained by applying the functor $\mathcal{F} \mapsto H^{i}\left(\mathbb{P}^{n}, \mathcal{F}\right)$ to the complex $\widetilde{F}(\boldsymbol{b})$, then we have $H^{0}\left(\mathbb{P}^{n}, \widetilde{F}(\boldsymbol{b})\right)=H^{0}\left(\mathbb{P}^{n}, \widetilde{G}(\boldsymbol{b})\right)$. The notation $H^{i}\left(\mathbb{P}^{n}, \widetilde{F}(\boldsymbol{b})\right)$ should not be confused with the hypercohomology group $\mathbb{H}^{i}\left(\mathbb{P}^{n}, \widetilde{F}(\boldsymbol{b})\right)$, which equals $H^{i}\left(\mathbb{P}^{n}, \widetilde{M}(\boldsymbol{b})\right)$ because $\widetilde{F}$ is a locally free resolution of the sheaf $\widetilde{M}$. Since $\mathbf{0} \in \operatorname{reg} M$ and $\boldsymbol{b} \geqslant \mathbf{0}$, the Hilbert polynomial and Hilbert function of $M$ agree in degree $\boldsymbol{b}$. Because $F$ is a minimal free resolution of $M$, it follows that the strand $[F]_{\boldsymbol{b}}:=\left[\left(F_{0}\right)_{\boldsymbol{b}} \longleftarrow\left(F_{1}\right)_{\boldsymbol{b}} \longleftarrow \cdots\right]$ is quasi-isomorphic to $M_{\boldsymbol{b}}$, and hence

$$
\mathbb{H}^{0}\left(\mathbb{P}^{n}, \widetilde{F}(\boldsymbol{b})\right)=M_{\boldsymbol{b}} \cong[F]_{\boldsymbol{b}}=H^{0}\left(\mathbb{P}^{n}, \widetilde{F}(\boldsymbol{b})\right) \cong H^{0}\left(\mathbb{P}^{\boldsymbol{n}}, \widetilde{G}(\boldsymbol{b})\right) .
$$

If the line bundle $\mathcal{O}_{\mathbb{P} \boldsymbol{n}}(-\boldsymbol{a}+\boldsymbol{n})$ has global sections, then we see that $\mathcal{O}_{\mathbb{P} \boldsymbol{n}}(-\boldsymbol{a}+\boldsymbol{b})$ has no higher cohomology. Therefore, the only summands in $\widetilde{F}$ that can potentially have higher cohomology are those that also appear in $\widetilde{E}$. Thus, for all $i>0$, we have $H^{i}\left(\mathbb{P}^{n}, \widetilde{F}(\boldsymbol{b})\right)=H^{i}\left(\mathbb{P}^{n}, \widetilde{E}(\boldsymbol{b})\right)$ and $H^{i}\left(\mathbb{P}^{n}, \widetilde{G}(\boldsymbol{b})\right)=0$. It follows that $\mathbb{H}^{0}\left(\mathbb{P}^{n}, \widetilde{G}(\boldsymbol{b})\right) \cong H^{0}\left(\mathbb{P}^{\boldsymbol{n}}, \widetilde{G}(\boldsymbol{b})\right)$ and $\mathbb{H}^{i}\left(\mathbb{P}^{n}, \widetilde{G}(\boldsymbol{b})\right)=0$ for all $i>0$. Hence, the long exact sequence in hypercohomology yields

$$
\mathbb{H}^{i}\left(\mathbb{P}^{\boldsymbol{n}}, \widetilde{E}(\boldsymbol{b})\right)= \begin{cases}0 & \text { if } i=0, \\ \mathbb{H}^{i}\left(\mathbb{P}^{\boldsymbol{n}}, \widetilde{F}(\boldsymbol{b})\right) & \text { if } i>0 .\end{cases}
$$

Since $\boldsymbol{b} \in \operatorname{reg} M$, the sheaf $\widetilde{M}(\boldsymbol{b})$ has no higher cohomology and $\widetilde{F}(\boldsymbol{b})$ has no higher hypercohomology. By Lemma 3.3, we conclude that $\widetilde{E}$ is quasi-isomorphic to 0. 


\section{VIRTUAL RESOLUTIONS}

Although Theorem 3.1 presents the virtual resolution of the pair $(M, \boldsymbol{d})$ as a subcomplex of a minimal free resolution, the following algorithm shows that we can compute a virtual resolution of the pair $(M, \boldsymbol{d})$ without first computing an entire minimal free resolution. Our approach is similar to that in [MS04, Theorem 1.5], which allows one to certify that an element belongs to the regularity of a module from just part of its minimal free resolution. Alternatively, one can verify that an element belongs to the regularity by using the Tate resolutions appearing in [EES15, Section 4]; the package TateOnProducts [EESS18] already implements these algorithms in Macaulay2 [GS19]. For a module $M$ and a degree $\boldsymbol{d} \in \mathbb{Z}^{r}$, let $M_{\leqslant \boldsymbol{d}}$ denote the submodule generated by $\bigoplus_{\boldsymbol{a} \leqslant \boldsymbol{d}} M_{\boldsymbol{a}}$.

Algorithm 3.4 (Computing virtual resolutions of a pair).

Input: $\quad$ A finitely generated $\mathbb{Z}^{r}$-graded $B$-saturated $S$-module $M$ and

a vector $\boldsymbol{d} \in \mathbb{Z}^{r}$ such that $\boldsymbol{d} \in \operatorname{reg} M$.

Output: The virtual resolution $G$ of the pair $(M, \boldsymbol{d})$.

Initialize $K:=M$ and $i:=0$;

While $K \neq 0$ do

Choose a homogeneous minimal set $\mathscr{G}$ of generators for $K$;

Initialize $G_{i}:=\bigoplus_{g \in \mathscr{G}} S(-\operatorname{deg}(g))$ and $\varphi_{i}: G_{i} \rightarrow K$ to be the corresponding surjection;

Set $K:=\left(\operatorname{Ker} \varphi_{i}\right) \leqslant \boldsymbol{d}+\boldsymbol{n}$;

Set $i:=i+1$;

Return $G:=\left[G_{0} \stackrel{\varphi_{1}}{\longleftarrow} G_{1} \stackrel{\varphi_{2}}{\longleftarrow} G_{2} \longleftarrow \cdots\right]$.

Proof of correctness. Let $G$ be the complex produced by the algorithm, let $F$ be the minimal free resolution of $M$, and let $G^{\prime}$ be the virtual resolution of $(M, \boldsymbol{d})$. Let $\varphi, \partial$, and $\psi$ be the differentials of $G, F$, and $G^{\prime}$, respectively. We have $G_{0}=F_{0}=G_{0}^{\prime}$, as $\boldsymbol{d} \in \operatorname{reg} M$ implies that $M$ is generated in degree at most $\boldsymbol{d}$ by [MS04, Theorem 1.3].

The definition of $G^{\prime}$ implies that $\left(\operatorname{Im} \partial_{i}\right)_{\leqslant \boldsymbol{d}+\boldsymbol{n}}$ equals $\operatorname{Im} \psi_{i}$. We use induction on $i$ to prove that $\operatorname{Im} \varphi_{i}=\left(\operatorname{Im} \partial_{i}\right)_{\leqslant \boldsymbol{d}+\boldsymbol{n}}$. When $i=1$, we have $\operatorname{Im} \varphi_{1}=\left(\operatorname{Im} \partial_{1}\right)_{\leqslant \boldsymbol{d}+\boldsymbol{n}}$. For $i>1$, the key observation is $\left(\operatorname{Im} \partial_{i}\right)_{\leqslant \boldsymbol{d}+\boldsymbol{n}}=\left(\operatorname{Ker} \partial_{i-1}\right)_{\leqslant \boldsymbol{d}+\boldsymbol{n}}=\left(\left.\operatorname{Ker} \partial_{i-1}\right|_{G_{i-1}}\right)_{\leqslant \boldsymbol{d}+\boldsymbol{n}}$, where the second equality holds because any element in $\left(\operatorname{Ker} \partial_{i-1}\right)_{\leqslant \boldsymbol{d}+\boldsymbol{n}}$ depends only on the restriction of $\partial_{i-1}$ to $G_{i-1}$. By induction, we have $\operatorname{Im} \varphi_{i-1}=\left(\operatorname{Im} \partial_{i-1}\right)_{\leqslant \boldsymbol{d}+\boldsymbol{n}}$, so $\left(\left.\operatorname{Ker} \partial_{i-1}\right|_{G_{i-1}}\right)_{\leqslant \boldsymbol{d}+\boldsymbol{n}}=\left(\operatorname{Ker} \varphi_{i-1}\right)_{\leqslant \boldsymbol{d}+\boldsymbol{n}}=\operatorname{Im} \varphi_{i}$. Therefore, we conclude that $\operatorname{Im} \varphi_{i}=\operatorname{Im} \psi_{i}$ for all $i$ and $G \cong G^{\prime}$.

Remark 3.5. Although Algorithm 3.4 bears a similarity with the linear resolutions considered in [EES15, Proposition 2.7], the free modules appearing in a given term of our virtual resolutions need not be generated in a single degree, and our complexes need not be acyclic.

The following example demonstrates that the virtual resolution of a pair does depend on the choice of element in the regularity.

Example 3.6. Let $Z \subset \mathbb{P}^{1} \times \mathbb{P}^{1} \times \mathbb{P}^{2}$ be the subscheme consisting of six general points, and let $I$ be the corresponding $B$-saturated $S$-ideal. Macaulay2 [GS19] shows that the minimal free resolution of $S / I$ has the form $S^{1} \longleftarrow S^{37} \longleftarrow S^{120} \longleftarrow S^{166} \longleftarrow S^{120} \longleftarrow S^{45} \longleftarrow S^{7} \longleftarrow$, where for brevity we have omitted the twists. Using [MS04, Proposition 6.7], it follows that, up to symmetry in the first two factors, the minimal elements in the regularity of $S / I$ are $(5,0,0),(2,1,0),(1,0,1)$, and $(0,0,2)$. Table 3.1 compares some basic numerical invariants for the minimal free resolution and the corresponding virtual resolutions. The total Betti numbers of a free complex $F$ are the 


\section{Berkesch, D. Erman and G.G. Smith}

TABLE 3.1. Comparison of various free complexes associated with $Z$

\begin{tabular}{llc}
\hline \multicolumn{1}{c}{ Type of free complex } & \multicolumn{1}{c}{ Total Betti numbers } & Number of twists \\
\hline minimal free resolution of $S / I$ & $(1,37,120,166,120,45,7)$ & 78 \\
virtual resolution of the pair $(S / I,(5,0,0))$ & $(1,24,50,33,6)$ & 18 \\
virtual resolution of the pair $(S / I,(2,1,0))$ & $(1,29,73,66,21)$ & 22 \\
virtual resolution of the pair $(S / I,(1,0,1))$ & $(1,25,63,57,18)$ & 15 \\
virtual resolution of the pair $(S / I,(0,0,2))$ & $(1,22,51,42,12)$ & 13 \\
\hline
\end{tabular}

ranks of the terms $F_{i}$ ignoring the twists. Since $Z$ has codimension 4 , part (i) of Proposition 2.5 implies that any virtual resolution for $S / I$ must have length at least 4 , so the minimum is achieved by all of these virtual resolutions. All four virtual resolutions also have a nonzero first homology module, which is supported on the irrelevant ideal. The first three virtual resolutions also have nonzero second homology modules. By examining the twists, we see that no pair of these virtual resolutions are comparable. This corresponds to the fact that $\operatorname{reg}(S / I)$ has several distinct minimal elements.

\section{Virtual resolutions for punctual schemes}

This section formulates and proves an extension of a property of points in projective space. While every punctual scheme in projective space is arithmetically Cohen-Macaulay, this fails when the ambient space is a product of projective spaces; the minimal free resolution is nearly always too long. However, by using virtual resolutions, we obtain a unexpected variant for points in $\mathbb{P}^{n}$.

To state this analogue, recall that the irrelevant ideal on $\mathbb{P}^{\boldsymbol{n}}$ is $B=\bigcap_{i=1}^{r}\left\langle x_{i, 0}, x_{i, 1}, \ldots, x_{i, n_{i}}\right\rangle$. For a vector $\boldsymbol{a} \in \mathbb{N}^{r}$, set $B^{\boldsymbol{a}}:=\bigcap_{i=1}^{r}\left\langle x_{i, 0}, x_{i, 1}, \ldots, x_{i, n_{i}}\right\rangle^{a_{i}}$. With this notation, we may easily choose a different algebra to represent the structure sheaf on our punctual subscheme. In contrast with the virtual resolutions in Section 3, the next theorem produces acyclic free complexes.

Theorem 4.1. If $Z \subset \mathbb{P}^{\boldsymbol{n}}$ is a zero-dimensional scheme and $I$ is the corresponding $B$-saturated $S$-ideal, then there exists an $\boldsymbol{a} \in \mathbb{N}^{r}$ with $a_{r}=0$ such that the minimal free resolution of $S /\left(I \cap B^{\boldsymbol{a}}\right)$ has length equal to $|\boldsymbol{n}|=\operatorname{dim} \mathbb{P}^{\boldsymbol{n}}$. Moreover, any $\boldsymbol{a} \in \mathbb{N}^{r}$ with $a_{r}=0$ and other entries sufficiently positive yields such a virtual resolution of $S / I$.

Proof of Theorem 1.5. Applying Theorem 4.1, it suffices to choose $Q=B^{\boldsymbol{a}}$ for any $\boldsymbol{a} \in \mathbb{N}^{r}$ with $a_{r}=0$ and other entries sufficiently positive.

While Theorem 4.1 establishes that for appropriate $\boldsymbol{a} \in \mathbb{N}^{r}$, the projective dimension of $S /\left(I \cap B^{\boldsymbol{a}}\right)$ equals the codimension of $Z$, this does not mean that the algebra $S /\left(I \cap B^{\boldsymbol{a}}\right)$ is Cohen-Macaulay; the ideal $I \cap B^{a}$ will often fail to be unmixed. For instance, on $\mathbb{P}^{2} \times \mathbb{P}^{2}$, the ideals $\left\langle x_{i, 0}, x_{i, 1}, x_{i, 2}\right\rangle$ for $1 \leqslant i \leqslant 2$ have codimension 3 whereas a zero-dimensional scheme $Z$ would have codimension 4 . Nevertheless, we do get Cohen-Macaulayness in one case.

Corollary 4.2. If $Z \subset \mathbb{P}^{1} \times \mathbb{P}^{1}$ is a zero-dimensional subscheme and $I$ is the corresponding $B$-saturated $S$-ideal, then there exists an ideal $Q$ whose radical is $\left\langle x_{1,0}, x_{1,1}\right\rangle$ such that

(i) the algebra $S /(I \cap Q)$ is Cohen-Macaulay, and

(ii) there exists an $(m+1) \times m$ matrix over $S$ whose maximal minors generate $I \cap Q$. 


\section{VIRTUAL RESOLUTIONS}

Proof. Theorem 4.1 yields an $\boldsymbol{a} \in \mathbb{N}^{r}$ such that $I \cap B^{\boldsymbol{a}}$ has projective dimension 2 . On $\mathbb{P}^{1} \times \mathbb{P}^{1}$, the irrelevant ideal $B$ also has codimension 2, so $S /\left(I \cap B^{\boldsymbol{a}}\right)$ has codimension 2. Thus, the algebra $S /\left(I \cap B^{a}\right)$ is Cohen-Macaulay. The second statement is an immediate consequence of the Hilbert-Burch theorem [Eis95, Theorem 20.15].

Remark 4.3. Although we focus on products of projective spaces, our proofs for both Theorem 4.1 and Corollary 4.2 can be adapted to hold in the more general context of iterated projective bundles. For instance, let $X$ be the Hirzebruch surface with Cox ring $S=\mathbb{k}\left[y_{0}, y_{1}, y_{2}, y_{3}\right]$, where the variables have degrees $(1,0),(1,0),(-2,1)$, and $(0,1)$, respectively. Let $Z \subset X$ be the scheme-theoretic intersection of $y_{0}^{5} y_{2}^{2}+y_{1} y_{3}^{2}$ and $y_{0} y_{1}+y_{2} y_{1}^{3}$. If $I$ is the $B$-saturated $S$-ideal of $Z$, then $S / I$ has projective dimension 3 and $S /\left(I \cap\left\langle y_{0}, y_{1}\right\rangle^{a}\right)$ has projective dimension 2 for any $a \geqslant 4$.

As with the proof of Theorem 3.1, we collect two lemmas before proving Theorem 4.1.

Lemma 4.4. If $Z \subset \mathbb{P}^{\boldsymbol{n}}$ is a zero-dimensional scheme and $I$ is the corresponding $B$-saturated $S$-ideal, then there exists an $\boldsymbol{a} \in \mathbb{Z}^{r}$ with $a_{r}=0$ such that the depth of $(S / I)_{\geqslant a}$ is $r$. Moreover, this holds for any $\boldsymbol{a} \in \mathbb{Z}^{r}$ with $a_{r}=0$ and other entries sufficiently positive.

Proof. Extending the ground field does not change the depth of a module, so we assume that $\mathbb{k}$ is an infinite field. Since $\operatorname{dim}(S / I)_{\geqslant a}=\operatorname{dim}(S / I)=r$, the depth of $S / I$ is bounded above by $r$. For each $1 \leqslant i \leqslant r$, choose a general linear element $\ell_{i}$ in $\left\langle x_{i, 0}, x_{i, 1}, \ldots, x_{i, n_{i}}\right\rangle$. We claim that the elements $\ell_{1}, \ell_{2}, \ldots, \ell_{r}$ form a regular sequence on $(S / I)_{\geqslant a}$.

Let $M:=\bigoplus_{\boldsymbol{b} \in \mathbb{N}^{r}} H^{0}\left(Z, \mathcal{O}_{Z}(\boldsymbol{b})\right)$. By construction, the elements $\ell_{1}, \ell_{2}, \ldots, \ell_{r}$ form a regular sequence on $M$. Since $I$ is $B$-saturated, it follows that $H_{B}^{0}(S / I)=0$. The exact sequence relating local cohomology and sheaf cohomology [CLS11, Theorem 9.5.7] gives

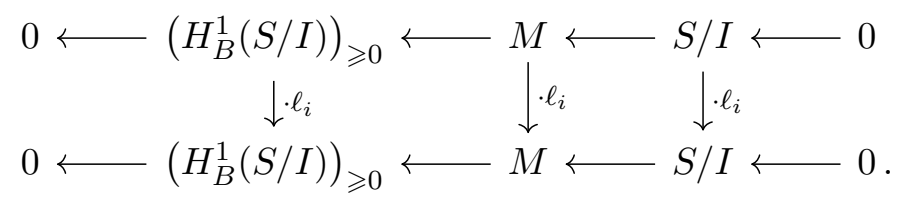

The middle vertical arrow is an isomorphism because $\ell_{i}$ does not vanish on any point in $Z$. Hence, the snake lemma [Eis95, Exercise A3.10] implies that the right vertical arrow is injective.

Focusing on the last component of $\mathbb{Z}^{r}$, we identify the Cox ring $R:=\mathbb{k}\left[x_{r, 0}, x_{r, 1}, \ldots, x_{r, n_{r}}\right]$ of the factor $\mathbb{P}^{n_{r}}$ with the subring $(S)_{(\mathbf{0}, *)}:=\bigoplus_{\alpha \in \mathbb{N}}(S)_{(\mathbf{0}, \alpha)}$ of $S$. For any $\boldsymbol{c}^{\prime} \in \mathbb{Z}^{r-1}$, consider the $R$-module $(S / I)_{\left(\boldsymbol{c}^{\prime}, *\right)}:=\bigoplus_{\alpha \in \mathbb{N}}(S)_{\left(\boldsymbol{c}^{\prime}, \alpha\right)}$. These modules form a directed set: for $\boldsymbol{c}^{\prime}, \boldsymbol{c}^{\prime \prime} \in \mathbb{Z}^{s-1}$ with $\boldsymbol{c}^{\prime \prime} \geqslant \boldsymbol{c}^{\prime}$, multiplication by the form $\ell_{1}^{\boldsymbol{c}_{1}^{\prime \prime}-\boldsymbol{c}_{1}^{\prime}} \ell_{2}^{\boldsymbol{c}_{2}^{\prime \prime}-\boldsymbol{c}_{2}^{\prime}} \cdots \ell_{s-1}^{\boldsymbol{c}_{s-1}^{\prime \prime}-\boldsymbol{c}_{s-1}^{\prime}}$ gives $(S / I)_{\left(\boldsymbol{c}^{\prime}, *\right)} \subseteq(S / I)_{\left(\boldsymbol{c}^{\prime \prime}, *\right)}$. Each $R$-module $(S / I)_{\left(\boldsymbol{c}^{\prime}, *\right)}$ is a submodule of $(M)_{\left(\boldsymbol{c}^{\prime}, *\right)}$, and $(M)_{\left(\boldsymbol{c}^{\prime}, *\right)} \cong(M)_{(0, *)}$. It follows that the $(S / I)_{\left(\boldsymbol{c}^{\prime}, *\right)}$ form an increasing sequence of finitely generated $R$-submodules of $(M)_{(0, *)}$, so this sequence stabilizes. In particular, if $\boldsymbol{a}^{\prime} \in \mathbb{Z}^{r-1}$ is sufficiently positive, then the inclusion $(S / I)_{\left(\boldsymbol{c}^{\prime}, *\right)} \subseteq(S / I)_{\left(\boldsymbol{c}^{\prime}+\boldsymbol{e}_{i}, *\right)}$ is an isomorphism for each $\boldsymbol{c}^{\prime} \geqslant \boldsymbol{a}^{\prime}$ and each $1 \leqslant i \leqslant r-1$. Hence, $\ell_{1}, \ell_{2}, \ldots, \ell_{r-1}$ form a regular sequence on $(S / I)_{\geqslant\left(\boldsymbol{a}^{\prime}, 0\right)}$, and

$$
\frac{(S / I)_{\geqslant\left(\boldsymbol{a}^{\prime}, 0\right)}}{\left\langle\ell_{1}, \ell_{2}, \ldots, \ell_{r-1}\right\rangle} \cong(S / I)_{\left(\boldsymbol{a}^{\prime}, *\right)} .
$$

Since the ideal $I$ is $B$-saturated, the homogeneous element $\ell_{r}$ is regular on $S / I$, so it is also regular on the $R$-module $(S / I)_{\left(\boldsymbol{a}^{\prime}, *\right)}$. Set $\boldsymbol{a}=\left(\boldsymbol{a}^{\prime}, 0\right)$; the Auslander-Buchsbaum formula [Eis95, Theorem 19.9] completes the proof. 


\section{Berkesch, D. Erman and G.G. Smith}

Remark 4.5. The proof of Lemma 4.4 shows that $(S / I)_{\geqslant a}$ has a multigraded regular sequence of length $r$, but neither $S /\left(I \cap B^{a}\right)$ nor $S / B^{a}$ generally has a multigraded regular sequence of length $r$.

LEMma 4.6. If $\boldsymbol{a}:=\left(a_{1}, a_{2}, \ldots, a_{i}, 0, \ldots, 0\right) \in \mathbb{Z}^{r}$ for some $1 \leqslant i \leqslant r$, then the projective dimension of $S / B^{a}$ is at most $n_{1}+n_{2}+\cdots+n_{i}+1$.

Proof. We proceed by induction on $i$. The case $i=1$ is just the Hilbert syzygy theorem [Eis95, Theorem 1.3] applied to the Cox ring of $\mathbb{P}^{n_{1}}$. When $i>1$, set $\boldsymbol{a}^{\prime}:=\left(a_{1}, a_{2}, \ldots, a_{i-1}, 0, \ldots, 0\right)$ and $\boldsymbol{a}^{\prime \prime}:=\left(0, \ldots, 0, a_{i}, 0, \ldots, 0\right)$, so that $\boldsymbol{a}=\boldsymbol{a}^{\prime}+\boldsymbol{a}^{\prime \prime}$ and $B^{\boldsymbol{a}}=B^{\boldsymbol{a}^{\prime}} \cap B^{\boldsymbol{a}^{\prime \prime}}$. The short exact sequence $0 \longleftarrow S /\left(B^{a^{\prime}}+B^{a^{\prime \prime}}\right) \longleftarrow S / B^{a^{\prime}} \longleftarrow S / B^{a} \longleftarrow 0$ yields

$$
\operatorname{pdim}\left(S / B^{\boldsymbol{a}}\right) \leqslant \max \left\{\operatorname{pdim}\left(S / B^{\boldsymbol{a}^{\prime}}\right), \operatorname{pdim}\left(S / B^{\boldsymbol{a}^{\prime \prime}}\right), \operatorname{pdim}\left(S /\left(B^{\boldsymbol{a}^{\prime}}+B^{\boldsymbol{a}^{\prime \prime}}\right)\right)-1\right\} .
$$

By induction, the projective dimension of $S / B^{a^{\prime}}$ is at most $n_{1}+n_{2}+\cdots+n_{i-1}+1$. By the Hilbert syzygy theorem on $\mathbb{P}^{n_{i}}$, the projective dimension of $S / B^{\boldsymbol{a}^{\prime \prime}}$ is at most $n_{i}+1$. Since $B^{\boldsymbol{a}^{\prime}}$ and $B^{\boldsymbol{a}^{\prime \prime}}$ are supported on disjoint sets of variables, $\operatorname{pdim}\left(S /\left(B^{\boldsymbol{a}^{\prime}}+B^{\boldsymbol{a}^{\prime \prime}}\right)\right)=\operatorname{pdim}\left(S / B^{\boldsymbol{a}^{\prime}}\right)+\operatorname{pdim}\left(S / B^{\boldsymbol{a}^{\prime \prime}}\right)$, which is at most $n_{1}+n_{2}+\cdots+n_{i}+2$. Applying (4.1), we obtain the result.

Proof of Theorem 4.1. Let $\boldsymbol{a} \in \mathbb{Z}^{r}$ with $a_{r}=0$ and other entries sufficiently positive. There is a short exact sequence $0 \longleftarrow S / B^{a} \longleftarrow S /\left(I \cap B^{a}\right) \longleftarrow(S / I)_{\geqslant a} \longleftarrow 0$. By Proposition 2.5, it suffices to prove that the projective dimension of $S /\left(I \cap B^{a}\right)$ is at most $n_{1}+n_{2}+\cdots+n_{r}$. Lemma 4.4 shows that $(S / I)_{\geqslant a}$ has projective dimension $n_{1}+n_{2}+\cdots+n_{r}$, and Lemma 4.6 shows that $S / B^{\boldsymbol{a}}$ has projective dimension at most $n_{1}+n_{2}+\cdots+n_{r-1}+1$. It follows that the projective dimension of $S /\left(I \cap B^{\boldsymbol{a}}\right)$ is at most $n_{1}+n_{2}+\cdots+n_{r}$ as well.

The next example compares the virtual resolutions produced by Theorems 1.3 and 1.5; neither seems to have a definitive advantage over the other.

Example 4.7. As in Example 3.6, let $Z \subset \mathbb{P}^{1} \times \mathbb{P}^{1} \times \mathbb{P}^{2}$ be the subscheme consisting of six general points, and let $I$ be the corresponding $B$-saturated $S$-ideal. Table 4.1 compares some basic numerical invariants for virtual resolutions arising from Theorem 4.1. Since the virtual resolutions in Table 4.1 involve nonminimal generators for $I$, they are different from those in Table 3.1. Conversely, the virtual resolutions appearing in Table 3.1 cannot be obtained from Theorem 1.5 because those free complexes are not acyclic.

TABLE 4.1. Comparison of various free complexes associated with $Z$

\begin{tabular}{llc}
\hline \multicolumn{1}{c}{ Type of free complex } & \multicolumn{1}{c}{ Total Betti numbers } & Number of twists \\
\hline minimal free resolution of $S / I$ & $(1,37,120,166,120,45,7)$ & 78 \\
virtual resolution from $\boldsymbol{a}=(2,1,0)$ & $(1,17,34,24,6)$ & 12 \\
virtual resolution from $\boldsymbol{a}=(3,3,0)$ & $(1,22,42,27,6)$ & 13 \\
\hline
\end{tabular}

We end this section by extending Corollary 4.2 to any smooth projective toric surface.

Proposition 4.8. Fix a smooth projective toric surface $X$. Let $Z \subset X$ be the subscheme consisting of $m$ general points, and let $I$ be the corresponding $B$-saturated $S$-ideal. There exists a virtual resolution $F:=\left[S \longleftarrow F_{1} \stackrel{\varphi}{\longleftarrow} F_{2} \longleftarrow 0\right]$ of $S / I$ such that $\operatorname{rank}\left(F_{1}\right)=$ $\operatorname{rank}\left(F_{2}\right)+1$, the maximal minors of $\varphi$ generate an ideal $J$ with $I=\left(J: B^{\infty}\right)$, and $S / J$ is Cohen-Macaulay. 


\section{VIRTUAL RESOLUTIONS}

Proof. Any smooth projective toric surface can be realized as a blowup $\pi: X \rightarrow Y$, where $Y$ is $\mathbb{P}^{2}$ or a Hirzebruch surface [CLS11, Theorem 10.4.3]. Since $\pi(Z) \subset Y$ is a punctual scheme, we can apply the Hilbert-Burch theorem when $Y$ is $\mathbb{P}^{2}$, or Corollary 4.2 and Remark 4.3 when $Y$ is a Hirzebruch surface, to obtain a resolution of $\mathcal{O}_{\pi(Z)}$ of the form $\mathcal{O}_{Y} \longleftarrow \mathcal{E}_{1} \longleftarrow \mathcal{E}_{2} \longleftarrow 0$, where $\mathcal{E}_{1}$ and $\mathcal{E}_{2}$ are sums of line bundles on $Y$. Our genericity hypothesis implies that $Z$ does not intersect the exceptional locus of $\pi$, so $\pi^{*} \mathcal{O}_{Y} \longleftarrow \pi^{*} \mathcal{E}_{1} \longleftarrow \pi^{*} \mathcal{E}_{2} \longleftarrow 0$ is a locally free resolution of $\mathcal{O}_{Z}$. The corresponding complex of $S$-modules is the desired virtual resolution for $S / I$.

Example 4.9. Consider the del Pezzo surface $X$ of degree 7 or, equivalently, the smooth Fano toric surface obtained by blowing up the projective plane at two torus-fixed points. The Cox ring of $X$ is $S:=\mathbb{k}\left[y_{0}, y_{1}, \ldots, y_{4}\right]$ equipped with the $\mathbb{Z}^{3}$-grading induced by

$$
\operatorname{deg}\left(y_{0}\right):=\left[\begin{array}{l}
1 \\
0 \\
0
\end{array}\right], \quad \operatorname{deg}\left(y_{1}\right):=\left[\begin{array}{r}
-1 \\
1 \\
0
\end{array}\right], \quad \operatorname{deg}\left(y_{2}\right):=\left[\begin{array}{r}
1 \\
-1 \\
1
\end{array}\right], \quad \operatorname{deg}\left(y_{3}\right):=\left[\begin{array}{r}
0 \\
1 \\
-1
\end{array}\right], \quad \operatorname{deg}\left(y_{4}\right):=\left[\begin{array}{l}
0 \\
0 \\
1
\end{array}\right] .
$$

With this choice of basis for $\operatorname{Pic}(X)$, the nef cone equals the positive orthant. Let $Z \subset X$ be the subscheme consisting of the three points $[1: 1: 1: 1: 1],[2: 1: 3: 1: 5]$, and $[7: 1: 11: 1: 13]$ (expressed in Cox coordinates), and let $I$ be the corresponding $B$-saturated $S$-ideal. Macaulay2 [GS19] shows that the minimal free resolution of $S / I$ has the form

$$
\begin{aligned}
& S(-1,0,-1)^{1} \quad S(0,-2,-2)^{1} \\
& \begin{array}{cc}
\oplus(0,-1,-1)^{1} & S(-1, \stackrel{\oplus}{\oplus},-1)^{2} \\
\oplus
\end{array}
\end{aligned}
$$

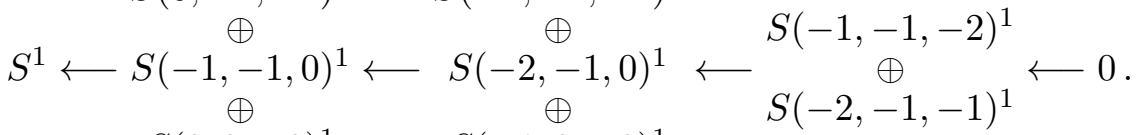

$$
\begin{aligned}
& S(0,0,-3)^{1} \quad S(-1,0,-3)^{1} \\
& S(-3,0,0)^{1} \quad S(-3,0,-1)^{1}
\end{aligned}
$$

However, there is a virtual resolution of $S / I$ having the form

$$
S^{1} \longleftarrow S(0,-2,0)^{3} \longleftarrow S(0,-3,0)^{2} \longleftarrow 0
$$

Example 4.10. Let $Z \subset \mathbb{P}^{1} \times \mathbb{P}^{1}$ be the subscheme consisting of $m$ general points, and let $I$ be the corresponding $B$-saturated $S$-ideal. Not only is there a Hilbert-Burch-type virtual resolution of $S / I$, it can be chosen to be a Koszul complex. Since $\operatorname{dim} H^{0}\left(\mathbb{P}^{1} \times \mathbb{P}^{1}, \mathcal{O}_{\mathbb{P}^{1} \times \mathbb{P}^{1}}(i, j)\right)=(i+1)(j+1)$, the generality of the points implies that $\operatorname{dim} H^{0}\left(\mathbb{P}^{1} \times \mathbb{P}^{1}, \mathcal{O}_{Z}(i, j)\right)=\min \{(i+1)(j+1), m\}$. Hence, if $m=2 k$ for some $k \in \mathbb{N}$, then two independent global sections of $\mathcal{O}_{\mathbb{P}^{1} \times \mathbb{P}^{1}}(1, k)$ vanish on $Z$. Using this pair, we obtain a virtual resolution of $S / I$ of the form $S \longleftarrow S(-1,-k)^{2} \longleftarrow S(-2,-2 k) \longleftarrow 0$. On the other hand, if $m=2 k+1$, then there are independent global sections of $\mathcal{O}_{\mathbb{P} 1 \times \mathbb{P} 1}(1, k)$ and $\mathcal{O}_{\mathbb{P}^{1} \times \mathbb{P}^{1}}(1, k+1)$ that vanish on $Z$, so we obtain a virtual resolution of $S / I$ having the form

$$
S \longleftarrow \underset{S(-1,-k-1)}{\oplus(-1,-k)} \longleftarrow S(-2,-2 k-1) \longleftarrow 0
$$

Question 4.11. Does Proposition 4.8 hold for any punctual scheme in a smooth toric surface? 


\section{Berkesch, D. Erman and G.G. Smith}

\section{Geometric applications}

In this section, we showcase four geometric applications of virtual resolutions. In particular, each of these support our overarching thesis that replacing minimal free resolutions by virtual resolutions yields the best geometric results for subschemes of $\mathbb{P}^{n}$.

\section{Unmixedness}

Given a subscheme that has a virtual resolution whose length equals its codimension, we prove an unmixedness result. Closely related to Proposition 2.5, this extends the classical unmixedness result for arithmetically Cohen-Macaulay subschemes; see [Eis95, Corollary 18.14].

Proposition 5.1. Let $Z \subset \mathbb{P}^{n}$ be a closed subscheme of codimension $c$, and let $I$ be the corresponding $B$-saturated $S$-ideal. If $S / I$ has a virtual resolution of length $c$, then every associated prime of I has codimension $c$.

Proof. Let $Q$ be an associated prime of $I$, and let $F$ denote a virtual resolution of $S / I$ having length $c$. Our hypothesis on $Z$ implies that $\operatorname{codim} Q \geqslant c$. Since $Q$ does not contain the irrelevant ideal $B$, localizing at $Q$ annihilates the homology of $F$ that is supported at $B$. Thus, the complex $F_{Q}$ is a free $S_{Q}$-resolution of $(S / I)_{Q}$. Since the projective dimension of a module is at least its codimension [Eis95, Proposition 18.2], it follows that $c \geqslant \operatorname{codim}_{S_{Q}}(S / I)_{Q}=\operatorname{codim} Q \geqslant c$.

\section{Deformation theory}

Using virtual resolutions, we generalize results about unobstructed deformations for arithmetically Cohen-Macaulay subschemes of codimension 2, arithmetically Gorenstein subschemes of codimension 3, and complete intersections. We first observe that the Piene-Schlessinger comparison theorem [PS85] applies more generally by relating the deformations of a closed subscheme $Y \subseteq \mathbb{P}^{n}$ with deformations of a corresponding graded module over the Cox ring.

Theorem 5.2. Let $Y \subset \mathbb{P}^{\boldsymbol{n}}$ be a closed subscheme, and let $I$ be a homogeneous $S$-ideal defining $Y$ scheme-theoretically and generated in degrees $\boldsymbol{d}_{1}, \boldsymbol{d}_{2}, \ldots, \boldsymbol{d}_{s}$. If the map $(S / I)_{\boldsymbol{d}_{i}} \rightarrow H^{0}\left(Y, \mathcal{O}_{Y}\left(\boldsymbol{d}_{i}\right)\right)$ is an isomorphism for all $1 \leqslant i \leqslant s$, then the embedded deformation theory of $Y \subset \mathbb{P}^{n}$ is equivalent to the degree zero embedded deformation theory of $\mathrm{V}(I) \subset \operatorname{Spec}(S)$.

Proof. Piene and Schlessinger's proof of the comparison theorem [PS85] goes through essentially verbatim by replacing projective space and its coordinate ring with $\mathbb{P}^{\boldsymbol{n}}$ and its Cox ring $S$.

Proof of Theorem 1.6. If $\boldsymbol{e} \in \operatorname{reg}(S / I)$ and $F$ is the virtual resolution of the pair $(S / I, \boldsymbol{e})$, then we have $\mathrm{H}_{0}(F)=S / J$ for some ideal $J$ whose $B$-saturation equals $I$. By Theorem 1.3 , the generating degrees for $J$ are a subset of those for $I$. It follows that $(S / J)_{\boldsymbol{d}}=H^{0}\left(Y, \mathcal{O}_{Y}(\boldsymbol{d})\right)$ for each degree $\boldsymbol{d}$ of a generator for $J$. Therefore, Theorem 5.2 implies that the embedded deformation theory of $Y$ is equivalent to the degree zero embedded deformation theory of the subscheme $\mathrm{V}(J) \subset \operatorname{Spec}(S)$.

(i) The virtual resolution $F$ has length 2 , so Proposition 2.5 implies that $F$ is the minimal free resolution of $S / J$. Thus, $S / J$ is Cohen-Macaulay of codimension 2, and [Art76, Section 5] or [Sch77] implies that its embedded deformations are unobstructed.

(ii) The virtual resolution $F$ has length 3 and $\min \left\{n_{i}+1: 1 \leqslant i \leqslant r\right\} \geqslant 3$, so Proposition 2.5 implies that $F$ is the minimal free resolution of $S / J$. Thus, $S / J$ is Gorenstein of codimension 3, and [Mir92, Theorem 2.1] implies that its embedded deformations are unobstructed.

(iii) Let $c:=\operatorname{codim} Y$. As $F$ is a Koszul complex, we have $F_{1}=\bigoplus_{i=1}^{c} S\left(-\boldsymbol{d}_{i}\right)$. Since $F$ is a virtual resolution of $S / I$, we also see that $\widetilde{J}$ equals the ideal sheaf $\mathcal{I}_{Y}$ for $Y \subset \mathbb{P}^{n}$. The complex 


\section{VIRTUAL RESOLUTIONS}

$\widetilde{F_{1}} \longleftarrow \widetilde{F_{2}} \longleftarrow \widetilde{F_{3}} \longleftarrow \cdots$ is a locally free resolution of $\mathcal{I}_{Y}$, so the normal bundle of $Y$ in $\mathbb{P}^{n}$ is $\mathcal{N}_{Y / \mathbb{P} n}:=\mathcal{H o m}\left(\mathcal{I}_{Y} / \mathcal{I}_{Y}^{2}, \mathcal{O}_{Y}\right) \cong \bigoplus_{i=1}^{c} \mathcal{O}_{Y}\left(\boldsymbol{d}_{i}\right)$. For a fixed deformation of $Y$, the obstruction is a Cech cocycle in $H^{1}\left(Y, \mathcal{N}_{Y / \mathbb{P} n}\right)$ determined by local lifts of the syzygies; see [Har10, Theorem 6.2]. However, since $Y$ is a scheme-theoretic complete intersection, its syzygies are all Koszul, so we can define this cocycle by lifting those Koszul syzygies globally on $Y$. Hence, the Čech cocycle in $H^{1}\left(Y, \mathcal{N}_{Y / \mathbb{P}^{n}}\right)$ is actually a coboundary, and the obstruction vanishes.

Remark 5.3. We suspect the hypothesis $\min \left\{n_{i}\right\} \geqslant 2$ in part (ii) of Theorem 1.6 is unnecessary.

Example 5.4. Consider the hyperelliptic curve $C \subset \mathbb{P}^{1} \times \mathbb{P}^{2}$ defined in Example 1.4. Applying part (i) of Theorem 1.6, we see that the virtual resolution from (1.2) implies that $C$ has unobstructed embedded deformations. Alternatively, this curve has a virtual resolution

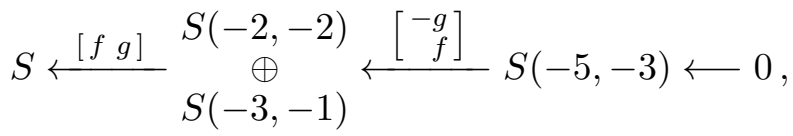

where $f=x_{1,0}^{2} x_{2,0}^{2}+x_{1,1}^{2} x_{2,1}^{2}+x_{1,0} x_{1,1} x_{2,2}^{2}$ and $g=x_{1,0}^{3} x_{2,2}+x_{1,1}^{3}\left(x_{2,0}+x_{2,1}\right)$, so part (iii) of Theorem 1.6 provides another proof that this curve has unobstructed embedded deformations.

\section{Regularity of tensor products}

Using virtual resolutions, we can prove bounds for the regularity of a tensor product, similar to the bounds obtained for projective space; see [Laz04, Proposition 1.8.8]. Let $\boldsymbol{e}_{i}$ denote the $i$ th standard basis vector in $\mathbb{Z}^{r}=\operatorname{Pic}\left(\mathbb{P}^{n}\right)$.

Proposition 5.5. Let $\mathcal{E}$ and $\mathcal{F}$ be coherent $\mathcal{O}_{\mathbb{P}^{n}}$-modules such that $\mathcal{T}_{\text {or }}{ }^{j}(\mathcal{E}, \mathcal{F})=0$ for all $j>0$. If $\boldsymbol{a} \in \operatorname{reg} \mathcal{E}$ and $\boldsymbol{b} \in \operatorname{reg} \mathcal{F}$, then we have $\boldsymbol{a}+\boldsymbol{b}+\mathbf{1}-\boldsymbol{e}_{i} \in \operatorname{reg}(\mathcal{E} \otimes \mathcal{F})$ for each $1 \leqslant i \leqslant r$.

Proof. Let $M$ and $N$ be the $B$-saturated $S$-modules corresponding to $\mathcal{E}$ and $\mathcal{F}$. Since $M(\boldsymbol{a})$ is 0-regular, Theorem 2.9 implies that it has a virtual resolution $F_{0} \longleftarrow F_{1} \longleftarrow \cdots$, where the degree of each generator of $F_{i}$ belongs to $\Delta_{i}+\mathbb{N}^{r}$. Similarly, $N(\boldsymbol{b})$ has a virtual resolution $G_{0} \longleftarrow G_{1} \longleftarrow \cdots$ satisfying the same conditions. The vanishing of Tor-groups implies that $H:=F \otimes G$ is a virtual resolution of $M(\boldsymbol{a}) \otimes N(\boldsymbol{b})$. Since $\Delta_{i}+\Delta_{j}+\mathbf{1}-\boldsymbol{e}_{i} \subseteq \Delta_{i+j}+\mathbb{N}^{r}$, it follows that the degree of each generator of the free module $H\left(\mathbf{1}-\boldsymbol{e}_{i}\right)_{k}$ belongs to $\Delta_{k}+\mathbb{N}^{r}$, for each $k$. Hence, Theorem 2.9 implies that $(M(\boldsymbol{a}) \otimes N(\boldsymbol{b}))\left(\mathbf{1}-\boldsymbol{e}_{i}\right)$ is $\mathbf{0}$-regular.

Remark 5.6. Proposition 5.5 is sharp. When $r=1$, it recovers [Laz04, Proposition 1.8.8], as the higher Tor-groups vanish whenever one of the two sheaves is locally free. If $r>1$, then it is possible to have $\mathbf{0}$-regular sheaves whose tensor product is not $\mathbf{0}$-regular. For instance, if $D, D^{\prime} \subset \mathbb{P}^{1} \times \mathbb{P}^{2}$ are degree $(1,1)$ hypersurfaces, then the product $\mathcal{O}_{D} \otimes \mathcal{O}_{D^{\prime}}$ is isomorphic to the structure sheaf $\mathcal{O}_{C}$ for a curve $C$ with $H^{1}\left(C, \mathcal{O}_{C}(0,-1)\right) \neq 0$.

\section{Vanishing of higher direct images}

A relative notion of Castelnuovo-Mumford regularity with respect to a given morphism is defined in terms of the vanishing of derived pushforwards; see [Laz04, Example 1.8.24]. Just as virtual resolutions yield sharper bounds on multigraded Castelnuovo-Mumford regularity, they also provide sharper bounds for the vanishing of derived pushforwards. For some $1 \leqslant s \leqslant r$, fix a subset $\left\{i_{1}, i_{2}, \ldots, i_{s}\right\} \subseteq\{1,2, \ldots, r\}$, and let $Y$ be the corresponding product $\mathbb{P}^{n_{i_{1}}} \times \mathbb{P}^{n_{i_{2}}} \times \cdots \times \mathbb{P}^{n_{i_{r}}}$ of projective spaces. The canonical projection $\pi: \mathbb{P}^{\boldsymbol{n}} \rightarrow Y$ induces an inclusion $\pi^{*}: \operatorname{Pic}(Y) \rightarrow$ $\operatorname{Pic}\left(\mathbb{P}^{\boldsymbol{n}}\right)$, and we write $\rho: \operatorname{Pic}\left(\mathbb{P}^{\boldsymbol{n}}\right) \rightarrow \operatorname{Coker}\left(\pi^{*}\right)=\operatorname{Pic}\left(\mathbb{P}^{\boldsymbol{n}}\right) / \operatorname{Pic}(Y)$. 


\section{Berkesch, D. Erman and G.G. Smith}

Proposition 5.7. Let $M$ be a finitely generated $\mathbb{Z}^{r}$-graded $S$-module, and consider $\boldsymbol{a} \in \mathbb{Z}^{r}$. If we have $\rho(\boldsymbol{a}) \in \rho(\operatorname{reg} M)$, then it follows that $\mathbf{R}^{i} \pi_{*} \widetilde{M}(\boldsymbol{a})=0$ for all $i>0$.

Proof. Since $\rho(\boldsymbol{a}) \in \rho(\operatorname{reg} M)$, we can choose a $\boldsymbol{b} \in \operatorname{Pic}(Y)$ such that $\boldsymbol{a}+\pi^{*} \boldsymbol{b} \in \operatorname{reg} M$. The projection formula [Har77, Exercise III.8.3] gives $\mathbf{R}^{i} \pi_{*} \widetilde{M}(\boldsymbol{a}) \otimes \mathcal{O}_{Y}(\boldsymbol{b})=\mathbf{R}^{i} \pi_{*} \widetilde{M}\left(\boldsymbol{a}+\pi^{*} \boldsymbol{b}\right)$ for all $\boldsymbol{b} \in \operatorname{Pic}(Y)$. Hence, by replacing $\boldsymbol{a}$ with $\boldsymbol{a}+\pi^{*} \boldsymbol{b}$, we assume that $\boldsymbol{a}$ itself lies in $\operatorname{reg} M$.

Let $G$ be the virtual resolution of the pair $(M, \boldsymbol{a})$, and consider a summand $S(-\boldsymbol{c})$ of $G$. By definition, we have $\boldsymbol{c} \leqslant \boldsymbol{a}+\boldsymbol{n}$. It follows that $-\boldsymbol{n} \leqslant-\boldsymbol{c}+\boldsymbol{a}$ and $\mathbf{R}^{i} \pi_{*} \mathcal{O}_{\mathbb{P} \boldsymbol{n}}(-\boldsymbol{c}+\boldsymbol{a})=0$ for all $i>0$. From the hypercohomology spectral sequence $E_{2}^{p, q}:=\mathbf{R}^{p} \widetilde{G_{-q}} \Longrightarrow \mathbf{R}^{p+q} \widetilde{M}$, we conclude that the higher direct images of $\widetilde{M}(\boldsymbol{a})$ also vanish.

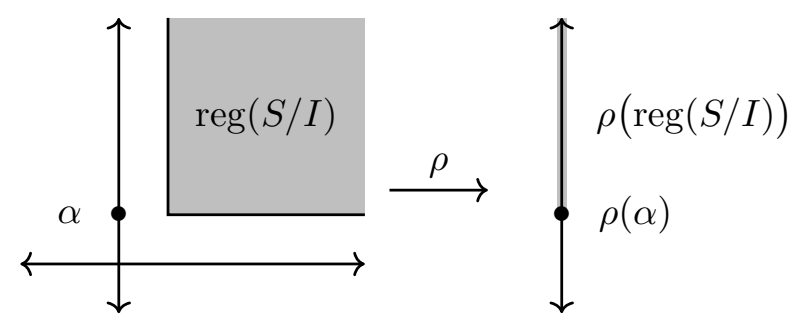

FiguRE 5.1. Representation of $\operatorname{reg}(S / I)$ and its image under $\rho$

Example 5.8. Let $Y \subset \mathbb{P}^{1} \times \mathbb{P}^{3}$ be the surface defined by the $B$-saturated ideal

$$
I=\left\langle\begin{array}{l}
x_{1,1} x_{2,1}^{2}+x_{1,0} x_{2,0} x_{2,2}+x_{1,1} x_{2,1} x_{2,3}, x_{1,0}^{2} x_{2,1}^{2}+x_{1,0} x_{1,1} x_{2,2}^{2}+x_{1,1}^{2} x_{2,0} x_{2,3}, \\
x_{1,0} x_{2,1}^{4}-x_{1,0} x_{2,0} x_{2,2}^{3}+x_{1,0} x_{2,1}^{3} x_{2,3}-x_{1,1} x_{2,0}^{2} x_{2,2} x_{2,3} \\
x_{2,1}^{6}-x_{2,0} x_{2,1}^{2} x_{2,2}^{3}+2 x_{2,1}^{5} x_{2,3}+x_{2,0}^{3} x_{2,2}^{2} x_{2,3}-x_{2,0} x_{2,1} x_{2,2}^{3} x_{2,3}+x_{2,1}^{4} x_{2,3}^{2}
\end{array}\right\rangle,
$$

and let $\pi: \mathbb{P}^{1} \times \mathbb{P}^{3} \rightarrow \mathbb{P}^{1}$ be the projection onto the first factor. To understand the vanishing of the higher direct images of $\mathcal{O}_{Y}$, we consider the minimal free resolution of $S / I$, which has the form

$$
\begin{aligned}
& S(-2,-2)^{1} \\
& \oplus \\
& S^{1} \longleftarrow \underset{S(-1,-4)^{1}}{\oplus(-1,-2)^{1}} \longleftarrow \underset{S(-1,-6)^{2}}{\oplus} \longleftarrow S(-2,-6)^{1} \longleftarrow 0 . \\
& S(0,-6)^{1}
\end{aligned}
$$

If we tensor the corresponding locally free resolution with the line bundle $\mathcal{O}_{Y}(0,3)$, then none of the terms in the resulting complex have nonzero higher direct images, so $\mathbf{R}^{1} \pi_{*} \mathcal{O}_{Y}(0, c)=0$ for $c \geqslant 3$. However, Proposition 5.7 yields a sharper vanishing result. Since Macaulay2 [GS19] shows that $(2,1) \in \operatorname{reg}(S / I)$, we have $\mathbf{R}^{1} \pi_{*} \mathcal{O}_{Y}(0, c)=0$ for all $c \geqslant 1$. This bound is sharp because a general fiber of $\pi$ is a curve of genus 1 .

\section{Questions}

We expect that virtual resolutions will produce further analogues of theorems involving minimal free resolutions on projective space. We close by highlighting several promising directions.

The first question is to find a notion of depth that controls the minimal length of a virtual resolution and provides an analogue of the Auslander-Buchsbaum theorem. 


\section{VIRTUAL RESOLUTIONS}

Question 6.1. Given an $S$-module $M$, what invariants of $M$ determine the length of the shortest possible virtual resolution of $M$ ?

Even understanding this question for curves in $\mathbb{P}^{1} \times \mathbb{P}^{2}$ would be compelling. In light of Theorem 1.6, this case would produce unirationality results for certain parameter spaces of curves.

Question 6.2. For what values of $d$, e, and $g$, does there exist a smooth curve in $\mathbb{P}^{1} \times \mathbb{P}^{2}$ of bidegree $(d, e)$ and genus $g$ with a virtual resolution of the form $S \longleftarrow F_{1} \longleftarrow F_{2} \longleftarrow 0$ ?

Proposition 5.1 and Theorem 1.6 suggest that having a virtual resolution whose length equals the codimension of the underlying variety can have significant geometric implications. As these results parallel the arithmetic Cohen-Macaulay property over projective space, it would be interesting to seek out analogues of being arithmetically Gorenstein.

Question 6.3. Consider a positive-dimensional subscheme $Z \subseteq \mathbb{P}^{n}$ such that $\omega_{Z}=\left.\mathcal{O}_{\mathbb{P} \boldsymbol{n}}(\boldsymbol{d})\right|_{Z}$ for some $\boldsymbol{d} \in \mathbb{Z}^{r}$. Is there a self-dual virtual resolution of $Z$ ?

It would also be interesting to better understand scheme-theoretic complete intersections.

Question 6.4. Develop an algorithm to determine whether a subvariety $Z \subseteq \mathbb{P}^{\boldsymbol{n}}$ has a virtual resolution that is a Koszul complex. This is already interesting in the case of points on $\mathbb{P}^{1} \times \mathbb{P}^{1}$.

Finally, we believe that many of these results should hold for more general toric varieties.

Question 6.5. Prove an analogue of Proposition 1.2 for an arbitrary smooth toric variety.

\section{ACKNOWLEDGEMENTS}

Some of this research was completed during visits to the Banff International Research Station (BIRS) and the Mathematical Sciences Research Institute (MSRI), and we are very grateful for their hospitality. We thank Lawrence Ein, David Eisenbud, Craig Huneke, Nathan Ilten, Rob Lazarsfeld, Mike Loper, Diane Maclagan, Frank-Olaf Schreyer, and Ian Shipman for helpful conversations. We also thank an anonymous referee for their valuable suggestions.

\section{REFERENCES}

Art76 M. Artin, Lectures on deformations of singularities, Lectures on Math. and Physics, vol. 54 (Tata Inst. Fund. Res., Bombay) 1976.

BC17 N. Botbol and M. Chardin, Castelnuovo Mumford regularity with respect to multigraded ideals, J. Algebra 474 (2017), 361-392; doi:10.1016/j.jalgebra.2016.11.017.

BS98 D. Bayer and B. Sturmfels, Cellular resolutions of monomial modules, J. reine angew. Math. 502 (1998), 123-140; doi:10.1515/crll.1998.083.

Căl05 A. Căldăraru, Derived categories of sheaves: a skimming, in Snowbird Lectures in Algebraic Geometry, Contemp. Math., vol. 388 (Amer. Math. Soc., Providence, RI, 2005), 43-75; doi: 10.1090/conm/388/07256.

CLS11 D. A. Cox, J. B. Little, and H. K. Schenck, Toric varieties, Grad. Stud. Math., vol. 124 (Amer. Math. Soc., Providence, RI, 2011); doi:10.1090/gsm/124.

Cox95 D. A. Cox, The homogeneous coordinate ring of a toric variety, J. Algebraic Geom. 4 (1995), no. $1,17-50$.

DFS16 A. Deopurkar, M. Fedorchuk, and D. Swinarski, Toward GIT stability of syzygies of canonical curves, Algebr. Geom. 3 (2016), no. 1, 1-22; doi:10.14231/AG-2016-001. 


\section{Berkesch, D. Erman and G.G. Smith}

DS00 W. Decker and F.-O. Schreyer, Non-general type surfaces in $\mathbf{P}^{4}$ : some remarks on bounds and constructions, J. Symbolic Comput. 29 (2000), no. 4-5, 545-582; doi:10.1006/jsco.1999. 0323.

EES15 D. Eisenbud, D. Erman, and F.-O. Schreyer, Tate resolutions for products of projective spaces, Acta Math. Vietnam. 40 (2015), no. 1, 5-36; doi:10.1007/s40306-015-0126-z.

EESS18 D. Eisenbud, D. Erman, F.-O. Schreyer, and M.E. Stillman, TateOnProducts, a Macaulay2 package, 2018, available at http://www.math.uiuc.edu/Macaulay2/.

Eis95 D. Eisenbud, Commutative algebra with a view toward algebraic geometry, Grad. Texts in Math., vol. 150 (Springer-Verlag, New York, 1995); doi:10.1007/978-1-4612-5350-1.

EL15 The gonality conjecture on syzygies of algebraic curves of large degree, Publ. Math. Inst. Hautes Études Sci. 122 (2015), 301-313; doi:10.1007/s10240-015-0072-2.

EP99 D. Eisenbud and S. Popescu, Gale duality and free resolutions of ideals of points, Invent. Math. 136 (1999), no. 2, 419-449; doi:10.1007/s002220050315.

Far09 G. Farkas, Koszul divisors on moduli spaces of curves, Amer. J. Math. 131 (2009), no. 3, 819-867; doi:10.1353/ajm.0.0053.

Fuj83 T. Fujita, Vanishing theorems for semipositive line bundles, Algebraic Geometry (Tokyo/Kyoto, 1982), Lecture Notes in Math., vol. 1016 (Springer, Berlin, 1983), 519-528; doi:10.1007/ BFb0099977.

GD61 A. Grothendieck and J. Dieudonné, Éléments de géométrie algébrique. III. Étude cohomologique des faisceaux cohérents. Première partie, Publ. Math. Inst. Hautes Études Sci. 11 (1961), 5-167; http://www . numdam. org/item?id=PMIHES_1961__11__5_0.

GGP95 A. V. Geramita, A. Gimigliano, and Y. Pitteloud, Graded Betti numbers of some embedded rational $n$-folds, Math. Ann. 301 (1995), no. 2, 363-380; doi:10.1007/BF01446634.

GP99 F. J. Gallego and B.P. Purnaprajna, Projective normality and syzygies of algebraic surfaces, J. reine angew. Math. 506 (1999), 145-180; doi:10.1515/crll.1999.506.145.

GR03 O. Gabber and L. Ramero, Almost ring theory, Lecture Notes in Math., vol. 1800 (Springer-Verlag, Berlin, 2003); doi:10.1007/b10047.

GS19 D. Grayson and M. Stillman, Macaulay2, a software system for research in algebraic geometry, 2019, available at https://faculty.math.illinois.edu/Macaulay2/.

GV15 E. Guardo and A. Van Tuyl, Arithmetically Cohen-Macaulay sets of points in $\mathbb{P}^{1} \times \mathbb{P}^{1}$, Springer Briefs Math. (Springer, Cham, 2015); doi:10.1007/978-3-319-24166-1.

Har77 R. Hartshorne, Algebraic geometry, Grad. Texts in Math., vol. 52 (Springer-Verlag, New YorkHeidelberg, 1977); doi:10.1007/978-1-4757-3849-0.

Har10 _ Deformation theory, Grad. Texts in Math., vol. 257 (Springer, New York, 2010); doi: 10.1007/978-1-4419-1596-2.

HH93 M. Hochster and C. Huneke, Phantom homology, Mem. Amer. Math. Soc. 103 (1993), no. 490; doi:10.1090/memo/0490.

Huy06 D. Huybrechts, Fourier-Mukai transforms in algebraic geometry, Oxford Math. Monogr. (The Clarendon Press, Oxford Univ. Press, Oxford, 2006); doi:10.1093/acprof : oso/9780199296866. 001.0001.

Laz04 R. Lazarsfeld, Positivity in algebraic geometry. I. classical setting: line bundles and linear series, Ergeb. Math. Grenzgeb. (3), vol. 48 (Springer-Verlag, Berlin, 2004); doi:10.1007/ 978-3-642-18808-4.

Mir92 R. M. Miró-Roig, Nonobstructedness of Gorenstein subschemes of codimension 3 in $\mathbf{P}^{n}$, Beitr. Algebra Geom. 33 (1992), 131-138.

MS04 D. Maclagan and G. G. Smith, Multigraded Castelnuovo-Mumford regularity, J. reine angew. Math. 571 (2004), 179-212; doi:10.1515/crll.2004.040.

PS85 R. Piene and M. Schlessinger, On the Hilbert scheme compactification of the space of twisted cubics, Amer. J. Math. 107 (1985), no. 4, 761-774; doi:10.2307/2374355. 


\section{VIRTUAL RESOLUTIONS}

Rob87 P. Roberts, Le théorème d'intersection, C. R. Acad. Sci. Paris Sér. I Math. 304 (1987), no. 7, 177-180.

Sch77 M. Schaps, Deformations of Cohen-Macaulay schemes of codimension 2 and non-singular deformations of space curves, Amer. J. Math. 99 (1977), no. 4, 669-685; doi:10.2307/2373859.

Voi02 C. Voisin, Green's generic syzygy conjecture for curves of even genus lying on a K3 surface, J. Eur. Math. Soc. 4 (2002), no. 4, 363-404; doi:10.1007/s100970200042.

Christine Berkesch cberkesc@math.umn.edu

School of Mathematics, University of Minnesota, Minneapolis, MN, 55455, USA

Daniel Erman derman@math.wisc.edu

Department of Mathematics, University of Wisconsin, Madison, WI, 53706, USA

Gregory G. Smith ggsmith@mast.queensu.ca

Department of Mathematics \& Statistics, Queen's University, Kingston, ON, K7L 3N6, Canada 